\title{
Industrial Espionage and Productivity
}

\author{
Albrecht Glitz Erik Meyersson \\ - Online Appendix -
}

Tables 


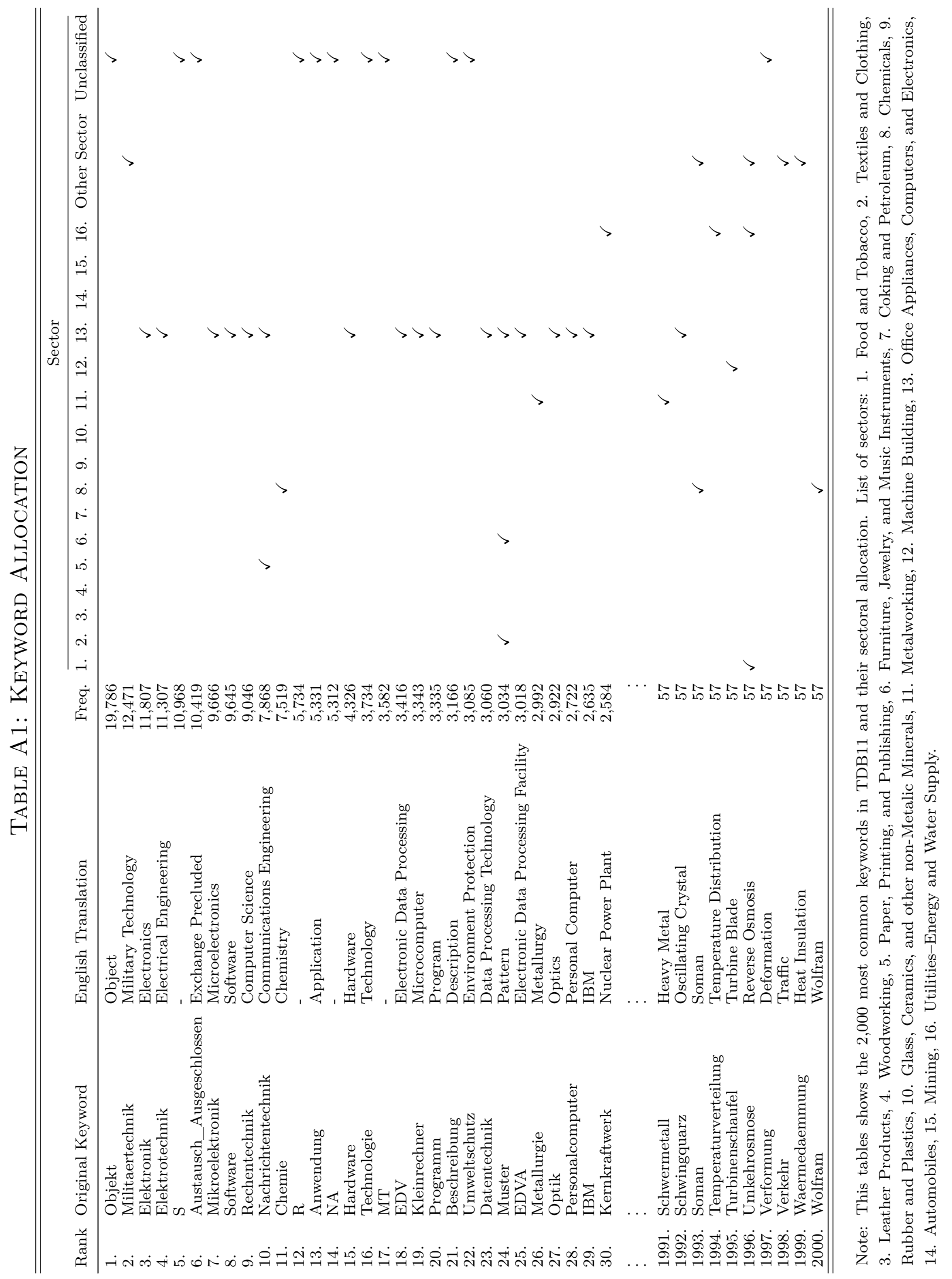


Table A2: Allocation Procedure - Examples

\begin{tabular}{llll}
\hline \hline ID & Keywords (Original) & Keywords (English) & Assigned Sector(s) \\
\hline Example 1: & & Chemicals, Electronics \\
2388127 & ELEKTROCHEMIE & Electrochemistry & Electronics \\
2388127 & OPTOELEKTRONIK & Optoelectronics & Electronics \\
2388127 & MIKROELEKTRONIK & Microelectronics & Electronics \\
2388127 & PLASMAPHYSIK & Plasma Physics & Chemicals \\
2388127 & OBJEKT & Object & Chemicals \\
2388127 & CHEMIE & Chemistry & \\
& Crystal Growing & \\
$\longrightarrow$ Baseline (unweighted): & Chemicals $\mathbf{1 ,}$ Electronics $\mathbf{1}$ & \\
\end{tabular}

\section{Example 2:}

$\begin{array}{lll}1674509 & \text { ELEKTROKERAMIK } & \text { Electrical Ceramic } \\ 1674509 & \text { KERAMIK } & \text { Ceramics } \\ 1674509 & \text { TITAN } & \text { Titan } \\ 1674509 & \text { PIEZOKERAMIK } & \text { Piezoceramics }\end{array}$

1674509 PIEZOKERAMIK

$\longrightarrow$ Baseline (unweighted):

$\longrightarrow$ Adjusted (weighted):

Glass, Ceramics, and Non-Metallic Minerals 1, Chemicals 1

Glass, Ceramics, and Non-Metallic Minerals 2/3, Chemicals 1/3

\section{Example 3:}

$\begin{array}{llll}2388709 & \text { OBJEKT } & \text { Object } & \\ 2388709 & \text { CHEMIE } & \text { Chemistry } & \text { Chemicals } \\ 2388709 & \text { AUSTAUSCH_AUSGESCHLOSSEN } & \text { Non-returnable } & \text { Networking } \\ 2388709 & \text { VERNETZUNG } & \text { Epoxy Resin } & \text { Chemicals, Rubber and Plastics, Electronics }\end{array}$

$\longrightarrow$ Baseline (unweighted):

Chemicals 1, Rubber and Plastics 1, Electronics 1

$\longrightarrow$ Adjusted (weighted):

Chemicals $1 / 2$, Rubber and Plastics $1 / 4$, Electronics $1 / 4$

\section{Example 4:}

\begin{tabular}{|c|c|c|c|}
\hline 2383885 & \multicolumn{2}{|l|}{ MASCHINENBAU } & Machine Building \\
\hline 2383885 & \multicolumn{2}{|c|}{ OBERFLAECHENBESCHICHTUNG } & Surface Coating \\
\hline 2383885 & \multicolumn{2}{|c|}{ HYDRAULIKANLAGE } & Hydraulic System \\
\hline 2383885 & \multicolumn{2}{|c|}{ FARBSPRITZROBOTER } & Painting Robot \\
\hline 2383885 & \multicolumn{2}{|l|}{ AUTOMOBILBAU } & Automobile Manufac \\
\hline 2383885 & \multicolumn{2}{|l|}{ LACKIERANLAGE } & Paint-spray Line \\
\hline 2383885 & \multicolumn{2}{|l|}{ MANIPULATOR } & Manipulator \\
\hline \multicolumn{2}{|c|}{$\begin{array}{l}\longrightarrow \text { Baseline (unweighted): } \\
\longrightarrow \text { Adjusted (weighted): }\end{array}$} & \multicolumn{2}{|c|}{$\begin{array}{l}\text { Machine Building } \mathbf{1} \text {, Motor Vehicle } \\
\text { Machine Building } 2 / 3 \text {, Motor Vehicles } 1\end{array}$} \\
\hline \multicolumn{4}{|c|}{ Example 5: } \\
\hline 1339125 & \multicolumn{2}{|l|}{ WEHRHYGIENE } & Military Hygiene \\
\hline 1339125 & \multicolumn{2}{|l|}{ WEHRMEDIZIN } & Military Medicine \\
\hline 1339125 & \multicolumn{2}{|c|}{ KOMBINATIONSSCHADEN } & Combined Handicaps \\
\hline 1339125 & \multicolumn{2}{|l|}{ THERAPIE } & Therapy \\
\hline 1339125 & \multicolumn{2}{|c|}{ INFEKTIONSPROPHYLAXE } & Infection Prophylaxis \\
\hline 1339125 & \multicolumn{2}{|l|}{ STRAHLENSCHUTZ } & Radiation Protection \\
\hline 1339125 & \multicolumn{2}{|l|}{ OBJEKT } & Object \\
\hline
\end{tabular}

$\longrightarrow$ Baseline (unweighted):

$\longrightarrow$ Adjusted (weighted):

$\begin{array}{lll}\text { Example } & \text { : } & \\ 1743402 & \text { BAGGER } & \text { Excavator } \\ 1743402 & \text { NA } & \text { NA } \\ 1743402 & \text { TAGEBAUAUSRUESTUNG } & \text { Open Pit Mining Equipment } \\ 1743402 & \text { KONSTRUKTION } & \text { Construction } \\ 1743402 & \text { SCHAUFELRADBAGGER } & \text { Bucket-wheel Excavator }\end{array}$

$\longrightarrow$ Baseline (unweighted):

$\longrightarrow$ Adjusted (weighted):

Note: This table provides six examples of how pieces of information are matched to sectors. Missing entries in the final column indicate that the corresponding keyword was either not classifiable (such as "Object" in example 1 or "Non-returnable" in example 3 ), not pertaining to any of the 16 sectors included in our analysis (such "Military Hygiene" in example 5), or not among the 2,000 most frequently occuring keywords in the database (such as "Electrical Ceramic" in example 2, "Painting Robot" in example 4, or "Excavator" in example 6). Examples 1 and 2 have good coverage as almost all keywords could be assigned to one or more sectors, examples 3 and 4 have moderate coverage, and examples 5 and 6 belong to the $18.6 \%$ of pieces of information that are not described by any sector-specific keyword and thus not accounted for in the empirical analysis. 
TABle A3: Top 20 Informants, 1968 - 1989

\begin{tabular}{|c|c|c|c|c|c|}
\hline Registration No. & Code Name & Pieces of Information & Reliability & First Active Year & Last Active Year \\
\hline$(1)$ & $(2)$ & $(3)$ & $(4)$ & $(5)$ & $(6)$ \\
\hline $\mathrm{XV} / 6603 / 80$ & FROEBEL & 5,344 & A & 1982 & 1989 \\
\hline $\mathrm{XV} / 2768 / 76$ & SEEMANN & 4,902 & $\mathrm{~A}$ & 1970 & 1988 \\
\hline $\mathrm{XV} / 1967 / 64$ & KOREN & 4,257 & A & 1973 & 1987 \\
\hline $\mathrm{XV} / 78 / 71$ & ZENTRUM & 3,373 & $\mathrm{~A}$ & 1969 & 1989 \\
\hline \multirow[t]{2}{*}{$\mathrm{XV} / 436 / 70$} & IRMGARD KRUEGER & 3,288 & $\mathrm{~A}$ & 1970 & 1989 \\
\hline & DR. GROSZ & 2,630 & $\mathrm{~A}$ & 1969 & 1974 \\
\hline $\mathrm{XV} / 1754 / 68$ & RING & 2,485 & A & 1968 & 1978 \\
\hline $\mathrm{XV} / 2550 / 74$ & HERZOG & 2,239 & A & 1974 & 1989 \\
\hline $\mathrm{XV} / 2234 / 74$ & JUERGEN & 1,631 & $\mathrm{~A}$ & 1969 & 1987 \\
\hline $\mathrm{XV} / 2110 / 67$ & OPTIK & 1,472 & A & 1969 & 1989 \\
\hline $\mathrm{XV} / 4070 / 70$ & LORENZ & 1,374 & B & 1971 & 1979 \\
\hline $\mathrm{XV} / 3074 / 78$ & SCHNEIDER & 1,347 & B & 1969 & 1989 \\
\hline $\mathrm{XV} / 6412 / 82$ & PICHLER & 1,157 & $\mathrm{~A}$ & 1982 & 1989 \\
\hline $\mathrm{XV} / 238 / 68$ & RITTER & 1,123 & B & 1969 & 1986 \\
\hline $\mathrm{XV} / 47 / 68$ & ERICH & 1,068 & $\mathrm{~A}$ & 1971 & 1988 \\
\hline $\mathrm{XV} / 450 / 86$ & ZELTER & 1,065 & B & 1984 & 1989 \\
\hline $\mathrm{XV} / 3 / 75$ & HARTMANN & 1,043 & A & 1969 & 1981 \\
\hline $\mathrm{XV} / 2001 / 73$ & JACK & 944 & $\mathrm{~A}$ & 1973 & 1987 \\
\hline XIV/14/69 & ALFRED & 890 & $\mathrm{~A}$ & 1970 & 1989 \\
\hline $\mathrm{XV} / 1508 / 75$ & WEBER & 867 & A & 1969 & 1980 \\
\hline
\end{tabular}

Note: Reliability is measured by the mode of the recorded assessments. An "A" denotes "reliable" (zuverlässig), a "B" denotes "trustworthy" (vertrauenswürdig), a "C" denotes "not checked" (nicht überprüft), a "D" denotes "questionable" (fragwürdig), and an "E" denotes "double agent" (Doppelagent). Only values A, B and C appear in the data. 
Table A4: Summary Statistics - By Sector

\begin{tabular}{|c|c|c|c|c|c|c|}
\hline & \multicolumn{2}{|c|}{ West Germany } & \multicolumn{2}{|c|}{ East Germany } & \multicolumn{2}{|c|}{ Difference } \\
\hline & Mean & $\mathrm{SD}$ & Mean & $\mathrm{SD}$ & Mean & $\mathrm{SD}$ \\
\hline & $(1)$ & $(2)$ & $(3)$ & $(4)$ & $(5)$ & (6) \\
\hline \multicolumn{7}{|l|}{ Food and Tobacco } \\
\hline Inflow /Y & & & 0.180 & $(0.020)$ & & \\
\hline Capital Share & 0.331 & $(0.025)$ & 0.436 & $(0.032)$ & & \\
\hline$\Delta \log \mathrm{TFP}$ & 0.036 & $(0.052)$ & 0.013 & $(0.058)$ & 0.023 & $(0.087)$ \\
\hline$\Delta$ Log Output per Worker & 0.052 & $(0.050)$ & 0.044 & $(0.043)$ & 0.008 & $(0.074)$ \\
\hline Patents/Y & 0.078 & $(0.013)$ & 0.016 & $(0.009)$ & 0.063 & $(0.021)$ \\
\hline Log TFP & 1.986 & $(0.085)$ & 1.588 & $(0.029)$ & 0.398 & $(0.091)$ \\
\hline Log Output per Worker & 3.428 & $(0.106)$ & 2.966 & $(0.051)$ & 0.463 & $(0.062)$ \\
\hline Imports/Y & 1.448 & $(0.158)$ & 0.147 & $(0.017)$ & 1.301 & $(0.147)$ \\
\hline \multicolumn{7}{|l|}{ Textiles and Clothing } \\
\hline Inflow/Y & & & 1.359 & $(0.306)$ & & \\
\hline Capital Share & 0.191 & $(0.041)$ & 0.208 & $(0.038)$ & & \\
\hline$\Delta \log \mathrm{TFP}$ & 0.075 & $(0.033)$ & 0.116 & $(0.057)$ & -0.041 & $(0.061)$ \\
\hline$\Delta$ Log Output per Worker & 0.090 & $(0.034)$ & 0.153 & $(0.057)$ & -0.063 & $(0.065)$ \\
\hline Patents/Y & 0.399 & $(0.046)$ & 0.169 & $(0.060)$ & 0.230 & $(0.098)$ \\
\hline Log TFP & 2.350 & $(0.113)$ & 0.272 & $(0.169)$ & 2.078 & $(0.065)$ \\
\hline Log Output per Worker & 3.099 & $(0.136)$ & 0.849 & $(0.221)$ & 2.250 & $(0.091)$ \\
\hline Imports/Y & 3.447 & $(0.736)$ & 1.097 & $(0.296)$ & 2.349 & $(0.749)$ \\
\hline \multicolumn{7}{|l|}{ Leather Products } \\
\hline Inflow $/ Y$ & & & 2.000 & $(0.184)$ & & \\
\hline Capital Share & 0.084 & $(0.032)$ & 0.208 & $(0.038)$ & & \\
\hline$\Delta \log \mathrm{TFP}$ & 0.045 & $(0.034)$ & -0.005 & $(0.075)$ & 0.050 & $(0.077)$ \\
\hline$\Delta$ Log Output per Worker & 0.053 & $(0.038)$ & 0.032 & $(0.059)$ & 0.021 & $(0.059)$ \\
\hline Patents/Y & 0.207 & $(0.041)$ & 0.232 & $(0.116)$ & -0.025 & $(0.082)$ \\
\hline Log TFP & 2.819 & $(0.062)$ & 0.800 & $(0.062)$ & 2.019 & $(0.106)$ \\
\hline Log Output per Worker & 3.123 & $(0.073)$ & 1.298 & $(0.048)$ & 1.825 & $(0.058)$ \\
\hline Imports/Y & 4.936 & $(1.509)$ & 0.700 & $(0.138)$ & 4.236 & $(1.445)$ \\
\hline \multicolumn{7}{|l|}{ Woodworking } \\
\hline Inflow/Y & & & 2.213 & $(0.416)$ & & \\
\hline Capital Share & 0.242 & $(0.030)$ & 0.348 & $(0.047)$ & & \\
\hline$\Delta \log \mathrm{TFP}$ & -0.006 & $(0.044)$ & 0.058 & $(0.060)$ & -0.063 & $(0.049)$ \\
\hline$\Delta$ Log Output per Worker & 0.004 & $(0.043)$ & 0.104 & $(0.093)$ & -0.100 & $(0.082)$ \\
\hline Patents/Y & 0.153 & $(0.031)$ & 0.065 & $(0.076)$ & 0.087 & $(0.058)$ \\
\hline Log TFP & 2.545 & $(0.034)$ & 0.380 & $(0.079)$ & 2.165 & $(0.095)$ \\
\hline Log Output per Worker & 3.523 & $(0.029)$ & 1.343 & $(0.158)$ & 2.181 & $(0.157)$ \\
\hline Imports/Y & 1.167 & $(0.262)$ & 0.272 & $(0.113)$ & 0.896 & $(0.283)$ \\
\hline \multicolumn{7}{|c|}{ Paper, Printing, and Publishing } \\
\hline Inflow/Y & & & 0.810 & $(0.144)$ & & \\
\hline Capital Share & 0.242 & $(0.030)$ & 0.405 & $(0.030)$ & & \\
\hline$\Delta \log \mathrm{TFP}$ & 0.018 & $(0.044)$ & 0.036 & $(0.028)$ & -0.018 & $(0.050)$ \\
\hline
\end{tabular}


Table A4 - continued from previous page

\begin{tabular}{|c|c|c|c|c|c|c|}
\hline & \multicolumn{2}{|c|}{ West Germany } & \multicolumn{2}{|c|}{ East Germany } & \multicolumn{2}{|c|}{ Difference } \\
\hline & Mean & $\mathrm{SD}$ & Mean & $\mathrm{SD}$ & Mean & $\mathrm{SD}$ \\
\hline & (1) & $(2)$ & $(3)$ & (4) & $(5)$ & (6) \\
\hline$\Delta$ Log Output per Worker & 0.043 & $(0.047)$ & 0.092 & $(0.024)$ & -0.049 & $(0.050)$ \\
\hline Patents/Y & 0.286 & $(0.034)$ & 0.024 & $(0.013)$ & 0.262 & $(0.044)$ \\
\hline Log TFP & 2.680 & $(0.041)$ & 1.763 & $(0.052)$ & 0.917 & $(0.031)$ \\
\hline Log Output per Worker & 3.672 & $(0.077)$ & 3.056 & $(0.125)$ & 0.616 & $(0.055)$ \\
\hline Imports/Y & 0.764 & $(0.129)$ & 0.122 & $(0.046)$ & 0.643 & $(0.125)$ \\
\hline \multicolumn{7}{|c|}{ Furniture, Jewelry, and Music Instruments } \\
\hline Inflow/Y & & & 2.936 & $(0.525)$ & & \\
\hline Capital Share & 0.115 & $(0.034)$ & 0.315 & $(0.042)$ & & \\
\hline$\Delta \log \mathrm{TFP}$ & 0.002 & $(0.047)$ & 0.064 & $(0.067)$ & -0.062 & $(0.092)$ \\
\hline$\Delta$ Log Output per Worker & 0.009 & $(0.044)$ & 0.106 & $(0.061)$ & -0.097 & $(0.077)$ \\
\hline Patents/Y & 0.153 & $(0.014)$ & 0.265 & $(0.165)$ & -0.112 & $(0.159)$ \\
\hline Log TFP & 3.264 & $(0.032)$ & 0.516 & $(0.114)$ & 2.748 & $(0.132)$ \\
\hline Log Output per Worker & 3.720 & $(0.030)$ & 1.452 & $(0.167)$ & 2.268 & $(0.170)$ \\
\hline Imports/Y & 0.932 & $(0.278)$ & 0.068 & $(0.015)$ & 0.864 & $(0.288)$ \\
\hline \multicolumn{7}{|l|}{ Coking and Petroleum } \\
\hline Inflow $/ Y$ & & & 0.230 & $(0.037)$ & & \\
\hline Capital Share & 0.618 & $(0.158)$ & 0.729 & $(0.068)$ & & \\
\hline$\Delta \log \mathrm{TFP}$ & -0.014 & $(0.119)$ & 0.065 & $(0.058)$ & -0.079 & $(0.134)$ \\
\hline$\Delta$ Log Output per Worker & 0.055 & $(0.188)$ & 0.144 & $(0.059)$ & -0.090 & $(0.170)$ \\
\hline Patents/Y & 0.246 & $(0.041)$ & 0.039 & $(0.008)$ & 0.207 & $(0.045)$ \\
\hline Log TFP & 1.136 & $(0.061)$ & 0.927 & $(0.112)$ & 0.209 & $(0.112)$ \\
\hline Log Output per Worker & 4.775 & $(0.156)$ & 4.592 & $(0.227)$ & 0.182 & $(0.152)$ \\
\hline Imports/Y & 18.242 & $(6.799)$ & 0.045 & $(0.019)$ & 18.197 & $(6.801)$ \\
\hline \multicolumn{7}{|l|}{ Chemicals } \\
\hline Inflow/Y & & & 2.205 & $(0.538)$ & & \\
\hline Capital Share & 0.321 & $(0.046)$ & 0.535 & $(0.028)$ & & \\
\hline$\Delta \log \mathrm{TFP}$ & 0.083 & $(0.081)$ & 0.063 & $(0.033)$ & 0.020 & $(0.094)$ \\
\hline$\Delta$ Log Output per Worker & 0.100 & $(0.086)$ & 0.128 & $(0.034)$ & -0.028 & $(0.086)$ \\
\hline Patents/Y & 1.034 & $(0.248)$ & 0.926 & $(0.205)$ & 0.108 & $(0.428)$ \\
\hline Log TFP & 2.160 & $(0.148)$ & 0.106 & $(0.096)$ & 2.054 & $(0.071)$ \\
\hline Log Output per Worker & 3.663 & $(0.175)$ & 2.473 & $(0.192)$ & 1.189 & $(0.051)$ \\
\hline Imports/Y & 2.202 & $(0.328)$ & 0.594 & $(0.152)$ & 1.608 & $(0.249)$ \\
\hline \multicolumn{7}{|l|}{ Rubber and Plastics } \\
\hline Inflow/Y & & & 2.969 & $(0.977)$ & & \\
\hline Capital Share & 0.315 & $(0.036)$ & 0.438 & $(0.046)$ & & \\
\hline$\Delta \log \mathrm{TFP}$ & 0.045 & $(0.053)$ & -0.022 & $(0.068)$ & 0.067 & $(0.071)$ \\
\hline$\Delta$ Log Output per Worker & 0.061 & $(0.051)$ & 0.058 & $(0.057)$ & 0.003 & $(0.063)$ \\
\hline Patents/Y & 0.353 & $(0.125)$ & 0.226 & $(0.083)$ & 0.127 & $(0.172)$ \\
\hline Log TFP & 2.310 & $(0.083)$ & 0.514 & $(0.068)$ & 1.796 & $(0.130)$ \\
\hline Log Output per Worker & 3.576 & $(0.107)$ & 2.093 & $(0.087)$ & 1.483 & $(0.046)$ \\
\hline Imports/Y & 0.504 & $(0.097)$ & 0.115 & $(0.030)$ & 0.389 & $(0.089)$ \\
\hline
\end{tabular}


Table A4 - continued from previous page

\begin{tabular}{|c|c|c|c|c|c|c|}
\hline & \multicolumn{2}{|c|}{ West Germany } & \multicolumn{2}{|c|}{ East Germany } & \multicolumn{2}{|c|}{ Difference } \\
\hline & Mean & $\mathrm{SD}$ & Mean & $\mathrm{SD}$ & Mean & $\mathrm{SD}$ \\
\hline & $(1)$ & $(2)$ & $(3)$ & $(4)$ & $(5)$ & $(6)$ \\
\hline \multicolumn{7}{|c|}{ Glass, Ceramics, and other Non-Metallic Minerals } \\
\hline Inflow/Y & & & 0.833 & $(0.215)$ & & \\
\hline Capital Share & 0.266 & $(0.038)$ & 0.434 & $(0.070)$ & & \\
\hline$\Delta \log \mathrm{TFP}$ & 0.044 & $(0.056)$ & 0.030 & $(0.117)$ & 0.015 & $(0.110)$ \\
\hline$\Delta$ Log Output per Worker & 0.072 & $(0.052)$ & 0.077 & $(0.126)$ & -0.005 & $(0.113)$ \\
\hline Patents/Y & 0.282 & $(0.038)$ & 0.123 & $(0.059)$ & 0.159 & $(0.088)$ \\
\hline Log TFP & 2.499 & $(0.068)$ & 0.692 & $(0.083)$ & 1.807 & $(0.095)$ \\
\hline Log Output per Worker & 3.670 & $(0.110)$ & 2.373 & $(0.123)$ & 1.297 & $(0.084)$ \\
\hline Imports/Y & 0.647 & $(0.110)$ & 0.055 & $(0.016)$ & 0.592 & $(0.097)$ \\
\hline \multicolumn{7}{|l|}{ Metalworking } \\
\hline Inflow/Y & & & 1.174 & $(0.318)$ & & \\
\hline Capital Share & 0.165 & $(0.040)$ & 0.339 & $(0.056)$ & & \\
\hline$\Delta \log \mathrm{TFP}$ & 0.043 & $(0.050)$ & 0.033 & $(0.057)$ & 0.011 & $(0.084)$ \\
\hline$\Delta$ Log Output per Worker & 0.050 & $(0.052)$ & 0.087 & $(0.054)$ & -0.037 & $(0.084)$ \\
\hline Patents/Y & 0.416 & $(0.032)$ & 0.236 & $(0.083)$ & 0.180 & $(0.112)$ \\
\hline Log TFP & 2.810 & $(0.072)$ & 0.279 & $(0.051)$ & 2.530 & $(0.064)$ \\
\hline Log Output per Worker & 3.522 & $(0.083)$ & 1.623 & $(0.141)$ & 1.899 & $(0.088)$ \\
\hline Imports/Y & 1.478 & $(0.192)$ & 0.816 & $(0.149)$ & 0.662 & $(0.146)$ \\
\hline \multicolumn{7}{|l|}{ Machine Building } \\
\hline Inflow/Y & & & 0.498 & $(0.144)$ & & \\
\hline Capital Share & 0.181 & $(0.030)$ & 0.278 & $(0.038)$ & & \\
\hline$\Delta \log \mathrm{TFP}$ & 0.024 & $(0.052)$ & 0.063 & $(0.042)$ & -0.039 & $(0.073)$ \\
\hline$\Delta$ Log Output per Worker & 0.036 & $(0.053)$ & 0.094 & $(0.052)$ & -0.058 & $(0.082)$ \\
\hline Patents/Y & 0.584 & $(0.063)$ & 0.472 & $(0.086)$ & 0.113 & $(0.139)$ \\
\hline Log TFP & 3.047 & $(0.053)$ & 1.687 & $(0.081)$ & 1.360 & $(0.045)$ \\
\hline Log Output per Worker & 3.735 & $(0.072)$ & 2.577 & $(0.120)$ & 1.158 & $(0.060)$ \\
\hline Imports/Y & 0.811 & $(0.160)$ & 0.219 & $(0.023)$ & 0.592 & $(0.146)$ \\
\hline \multicolumn{7}{|c|}{ Office Appliances, Computers, and Electronics } \\
\hline Inflow/Y & & & 5.339 & $(0.611)$ & & \\
\hline Capital Share & 0.264 & $(0.022)$ & 0.385 & $(0.051)$ & & \\
\hline$\Delta \log \mathrm{TFP}$ & 0.081 & $(0.034)$ & 0.046 & $(0.056)$ & 0.035 & $(0.066)$ \\
\hline$\Delta$ Log Output per Worker & 0.113 & $(0.043)$ & 0.110 & $(0.061)$ & 0.003 & $(0.081)$ \\
\hline Patents/Y & 1.453 & $(0.309)$ & 1.684 & $(0.272)$ & -0.231 & $(0.530)$ \\
\hline Log TFP & 2.412 & $(0.137)$ & 0.295 & $(0.061)$ & 2.117 & $(0.088)$ \\
\hline Log Output per Worker & 3.438 & $(0.191)$ & 1.466 & $(0.146)$ & 1.972 & $(0.057)$ \\
\hline Imports/Y & 1.000 & $(0.175)$ & 0.214 & $(0.072)$ & 0.786 & $(0.153)$ \\
\hline \multicolumn{7}{|l|}{ Motor Vehicles } \\
\hline Inflow/Y & & & 1.208 & $(0.383)$ & & \\
\hline Capital Share & 0.297 & $(0.038)$ & 0.433 & $(0.090)$ & & \\
\hline$\Delta \log$ TFP & 0.020 & $(0.055)$ & 0.028 & $(0.053)$ & -0.009 & $(0.078)$ \\
\hline$\Delta$ Log Output per Worker & 0.047 & $(0.054)$ & 0.108 & $(0.053)$ & -0.061 & $(0.086)$ \\
\hline Patents/Y & 0.396 & $(0.060)$ & 0.408 & $(0.090)$ & -0.012 & $(0.120)$ \\
\hline
\end{tabular}


Table A4 - continued from previous page

\begin{tabular}{|c|c|c|c|c|c|c|}
\hline & \multicolumn{2}{|c|}{ West Germany } & \multicolumn{2}{|c|}{ East Germany } & \multicolumn{2}{|c|}{ Difference } \\
\hline & Mean & SD & Mean & $\mathrm{SD}$ & Mean & $\mathrm{SD}$ \\
\hline & (1) & $(2)$ & $(3)$ & $(4)$ & $(5)$ & $(6)$ \\
\hline Log TFP & 2.545 & $(0.054)$ & 0.661 & $(0.041)$ & 1.884 & $(0.041)$ \\
\hline Log Output per Worker & 3.819 & $(0.094)$ & 2.041 & $(0.149)$ & 1.778 & $(0.069)$ \\
\hline Imports/Y & 1.147 & $(0.216)$ & 0.355 & $(0.046)$ & 0.792 & $(0.231)$ \\
\hline \multicolumn{7}{|l|}{ Mining } \\
\hline Inflow/Y & & & 0.177 & $(0.052)$ & & \\
\hline Capital Share & 0.277 & $(0.088)$ & 0.281 & $(0.102)$ & & \\
\hline$\Delta \log$ TFP & -0.047 & $(0.060)$ & 0.014 & $(0.054)$ & -0.061 & $(0.089)$ \\
\hline$\Delta$ Log Output per Worker & -0.028 & $(0.060)$ & 0.043 & $(0.049)$ & -0.071 & $(0.087)$ \\
\hline Patents/Y & 0.146 & $(0.035)$ & 0.080 & $(0.024)$ & 0.066 & $(0.032)$ \\
\hline Log TFP & 2.326 & $(0.069)$ & 2.073 & $(0.055)$ & 0.253 & $(0.118)$ \\
\hline Log Output per Worker & 3.704 & $(0.048)$ & 3.374 & $(0.093)$ & 0.330 & $(0.132)$ \\
\hline Imports/Y & 1.188 & $(0.277)$ & 0.041 & $(0.018)$ & 1.147 & $(0.266)$ \\
\hline \multicolumn{7}{|c|}{ Utilities - Energy and Water Supply } \\
\hline Inflow/Y & & & 0.260 & $(0.061)$ & & \\
\hline Capital Share & 0.602 & $(0.030)$ & 0.612 & $(0.045)$ & & \\
\hline$\Delta \log$ TFP & -0.000 & $(0.114)$ & 0.008 & $(0.070)$ & -0.008 & $(0.152)$ \\
\hline$\Delta$ Log Output per Worker & 0.045 & $(0.113)$ & 0.066 & $(0.067)$ & -0.021 & $(0.149)$ \\
\hline Patents/Y & 0.083 & $(0.015)$ & 0.042 & $(0.012)$ & 0.041 & $(0.026)$ \\
\hline Log TFP & 0.696 & $(0.075)$ & 1.191 & $(0.056)$ & -0.495 & $(0.112)$ \\
\hline Log Output per Worker & 4.393 & $(0.069)$ & 4.587 & $(0.125)$ & -0.194 & $(0.128)$ \\
\hline Imports/Y & 0.083 & $(0.011)$ & 0.000 & $(0.000)$ & 0.083 & $(0.011)$ \\
\hline
\end{tabular}

Note: Summary statistics computed for 3-year overlapping observations for the period 1970 to 1989 apart from the capital shares for East Germany which are based on the period 1995 to 2006 and refer to the aggregate capital shares in the ten new EU member states who joined in May 2004. Imports are cumulated over the last 3 years and measured in million dollars at constant 1995 prices. Output is measured in million euros at constant 1995 prices. Workers are measured in 1,000 so that output per worker is measured in 1,000 euros at constant 1995 prices. The number of observations is 15 for each industry (20 for the West German capital share, 12 for the East German capital share). 
TABle A5: Non-OVerlapping Observations

\begin{tabular}{|c|c|c|c|c|c|c|}
\hline & \multicolumn{3}{|c|}{ Log TFP } & \multicolumn{3}{|c|}{ Log Output per Worker } \\
\hline & $\begin{array}{l}\text { Baseline } \\
\text { spec }\end{array}$ & $\begin{array}{l}\text { Patents } \\
\text { gap }\end{array}$ & $\begin{array}{c}\text { Lagged } \\
\text { gap }\end{array}$ & $\begin{array}{c}\text { Baseline } \\
\text { spec }\end{array}$ & $\begin{array}{c}\text { Patents } \\
\text { gap }\end{array}$ & $\begin{array}{l}\text { Lagged } \\
\text { gap }\end{array}$ \\
\hline & $(1)$ & $(2)$ & $(3)$ & $(4)$ & $(5)$ & $(6)$ \\
\hline Espionage & $\begin{array}{l}-0.029 \\
(0.023)\end{array}$ & $\begin{array}{l}-0.041 \\
(0.023)\end{array}$ & $\begin{array}{l}-0.053 \\
(0.016)\end{array}$ & $\begin{array}{l}-0.026 \\
(0.020)\end{array}$ & $\begin{array}{l}-0.041 \\
(0.022)\end{array}$ & $\begin{array}{l}-0.042 \\
(0.022)\end{array}$ \\
\hline Patents Gap & & $\begin{array}{c}0.105 \\
(0.030)\end{array}$ & $\begin{array}{l}-0.006 \\
(0.035)\end{array}$ & & $\begin{array}{c}0.137 \\
(0.032)\end{array}$ & $\begin{array}{c}0.045 \\
(0.042)\end{array}$ \\
\hline Log TFP Gap & & & $\begin{array}{l}-0.544 \\
(0.128)\end{array}$ & & & \\
\hline Log Output/Worker Gap & & & & & & $\begin{array}{l}-0.478 \\
(0.139)\end{array}$ \\
\hline $\mathrm{P}$-value WB & 0.405 & 0.264 & 0.050 & 0.363 & 0.194 & 0.269 \\
\hline R-squared & 0.36 & 0.41 & 0.59 & 0.32 & 0.40 & 0.54 \\
\hline Observations & 80 & 80 & 80 & 80 & 80 & 80 \\
\hline
\end{tabular}

Note: Sample based on 3-year intervals and non-overlapping observations for the years 1973, 1976, 1979, 1982, and 1985. All regressions include time- and sector-specific fixed effects. Observations are weighted by the average number of workers in a sector. The dependent variable is the change in the log TFP gap between West and East Germany over the period t to $t+3$ in columns (1) to (3) and the change in the log output per worker gap over the period t to $t+3$ in columns (4) to (6). Standard errors are clustered at the sectoral level and shown in parentheses. P-value WB denotes p-values, relating to the espionage estimate, from Cameron et al. (2008)'s wild cluster bootstrap-t procedure using 999 replications. 
Table A6: Decompositions - Log Output Per Worker

\begin{tabular}{|c|c|c|c|c|c|c|}
\hline & \multicolumn{3}{|c|}{ Baseline Decomposition } & \multicolumn{3}{|c|}{ Flexible Decomposition } \\
\hline & FRG/GDR & FRG & GDR & FRG/GDR & FRG & GDR \\
\hline & (1) & (2) & (3) & $(4)$ & (5) & (6) \\
\hline \multicolumn{7}{|l|}{$\underline{\text { OLS }}$} \\
\hline Espionage & $\begin{array}{l}-0.039 \\
(0.017)\end{array}$ & $\begin{array}{l}-0.008 \\
(0.006)\end{array}$ & $\begin{array}{c}0.031 \\
(0.013)\end{array}$ & $\begin{array}{l}-0.020 \\
(0.015)\end{array}$ & $\begin{array}{l}-0.014 \\
(0.005)\end{array}$ & $\begin{array}{c}0.031 \\
(0.013)\end{array}$ \\
\hline Patents Gap & $\begin{array}{c}0.012 \\
(0.028)\end{array}$ & $\begin{array}{c}0.024 \\
(0.018)\end{array}$ & $\begin{array}{c}0.012 \\
(0.026)\end{array}$ & & & \\
\hline Log Output/Worker Gap & $\begin{array}{l}-0.514 \\
(0.100)\end{array}$ & $\begin{array}{l}-0.186 \\
(0.066)\end{array}$ & $\begin{array}{c}0.328 \\
(0.074)\end{array}$ & $\begin{array}{l}-0.563 \\
(0.116)\end{array}$ & & $\begin{array}{c}0.313 \\
(0.058)\end{array}$ \\
\hline GDR Patents/Y & & & & $\begin{array}{l}-0.145 \\
(0.067)\end{array}$ & & $\begin{array}{c}0.013 \\
(0.039)\end{array}$ \\
\hline FRG Patents/Y & & & & $\begin{array}{l}-0.111 \\
(0.095)\end{array}$ & $\begin{array}{c}0.086 \\
(0.025)\end{array}$ & \\
\hline P-value WB & 0.125 & 0.242 & 0.110 & 0.165 & 0.022 & 0.084 \\
\hline R-squared & 0.51 & 0.64 & 0.41 & 0.53 & 0.60 & 0.41 \\
\hline Observations & 240 & 240 & 240 & 240 & 240 & 240 \\
\hline \multicolumn{7}{|l|}{ IV - Old Informants } \\
\hline Espionage & $\begin{array}{l}-0.059 \\
(0.028)\end{array}$ & $\begin{array}{l}-0.033 \\
(0.019)\end{array}$ & $\begin{array}{c}0.025 \\
(0.017)\end{array}$ & $\begin{array}{l}-0.003 \\
(0.039)\end{array}$ & $\begin{array}{l}-0.044 \\
(0.019)\end{array}$ & $\begin{array}{c}0.035 \\
(0.019)\end{array}$ \\
\hline Patents Gap & $\begin{array}{c}0.018 \\
(0.025)\end{array}$ & $\begin{array}{c}0.031 \\
(0.022)\end{array}$ & $\begin{array}{c}0.013 \\
(0.021)\end{array}$ & & & \\
\hline Log Output/Worker Gap & $\begin{array}{l}-0.514 \\
(0.096)\end{array}$ & $\begin{array}{l}-0.185 \\
(0.064)\end{array}$ & $\begin{array}{c}0.328 \\
(0.065)\end{array}$ & $\begin{array}{l}-0.573 \\
(0.096)\end{array}$ & & $\begin{array}{c}0.314 \\
(0.056)\end{array}$ \\
\hline GDR Patents/Y & & & & $\begin{array}{l}-0.167 \\
(0.083)\end{array}$ & & $\begin{array}{c}0.012 \\
(0.038)\end{array}$ \\
\hline FRG Patents/Y & & & & $\begin{array}{l}-0.138 \\
(0.100)\end{array}$ & $\begin{array}{c}0.111 \\
(0.040)\end{array}$ & \\
\hline P-value WB & 0.354 & 0.308 & 0.426 & 0.952 & 0.116 & 0.319 \\
\hline F-stat & 60.8 & 60.8 & 60.8 & 13.8 & 14.1 & 77.4 \\
\hline Observations & 240 & 240 & 240 & 240 & 240 & 240 \\
\hline \multicolumn{7}{|l|}{ IV - Exit of Informants } \\
\hline Espionage & $\begin{array}{l}-0.119 \\
(0.040)\end{array}$ & $\begin{array}{l}-0.034 \\
(0.020)\end{array}$ & $\begin{array}{c}0.085 \\
(0.028)\end{array}$ & $\begin{array}{l}-0.094 \\
(0.040)\end{array}$ & $\begin{array}{l}-0.026 \\
(0.018)\end{array}$ & $\begin{array}{c}0.085 \\
(0.033)\end{array}$ \\
\hline Patents Gap & $\begin{array}{c}0.001 \\
(0.049)\end{array}$ & $\begin{array}{c}0.023 \\
(0.028)\end{array}$ & $\begin{array}{c}0.022 \\
(0.033)\end{array}$ & & & \\
\hline Log Output/Worker Gap & $\begin{array}{l}-0.613 \\
(0.133)\end{array}$ & $\begin{array}{l}-0.265 \\
(0.095)\end{array}$ & $\begin{array}{c}0.348 \\
(0.092)\end{array}$ & $\begin{array}{l}-0.645 \\
(0.140)\end{array}$ & & $\begin{array}{c}0.334 \\
(0.081)\end{array}$ \\
\hline GDR Patents/Y & & & & $\begin{array}{l}-0.079 \\
(0.085)\end{array}$ & & $\begin{array}{l}-0.017 \\
(0.061)\end{array}$ \\
\hline FRG Patents/Y & & & & $\begin{array}{l}-0.086 \\
(0.119)\end{array}$ & $\begin{array}{c}0.122 \\
(0.045)\end{array}$ & \\
\hline $\mathrm{P}$-value WB & 0.188 & 0.179 & 0.237 & 0.113 & 0.202 & 0.312 \\
\hline F-stat & 72.6 & 72.6 & 72.6 & 47.8 & 70.8 & 48.8 \\
\hline Observations & 192 & 192 & 192 & 192 & 192 & 192 \\
\hline
\end{tabular}

Sample based on 3-year intervals and overlapping observations for the period 1970 to 1989. All regressions include time- and sector-specific fixed effects. Observations are weighted by the average number of workers in a sector. The dependent variable is the change in the $\log$ output per worker gap between West and East Germany over the period $t$ and $t+3$ in columns (1) and (4) and the change in log output per worker between $t$ and $t+3$ in West and East Germany in columns (2) and (5) and (3) and (6), respectively. The instrumental variables are described in Section V A. Standard errors are clustered at the sectoral level and shown in parentheses. P-value WB denotes p-values, relating to the espionage estimate, from Cameron et al. (2008)'s wild cluster bootstrap-t procedure using 999 replications. 
TABle A7: Robustness - Log Output Per Worker

\begin{tabular}{|c|c|c|c|c|c|c|c|c|}
\hline & $\begin{array}{l}\text { Main } \\
\text { spec }\end{array}$ & $\begin{array}{l}\text { Weighted } \\
\text { by output }\end{array}$ & $\begin{array}{c}\text { No } \\
\text { weights }\end{array}$ & $\begin{array}{l}\text { No } \\
\text { IT }\end{array}$ & $\begin{array}{l}\text { Sector } \\
\text { trends }\end{array}$ & $\begin{array}{c}\text { Trade } \\
\text { gap }\end{array}$ & $\begin{array}{l}\text { Keyword } \\
\text { weighted }\end{array}$ & $\begin{array}{l}\text { Machine } \\
\text { learning }\end{array}$ \\
\hline & $(1)$ & $(2)$ & $(3)$ & (4) & $(5)$ & $(6)$ & $(7)$ & $(8)$ \\
\hline \multicolumn{9}{|l|}{ OLS } \\
\hline Espionage & $\begin{array}{l}-0.039 \\
(0.017)\end{array}$ & $\begin{array}{l}-0.070 \\
(0.035)\end{array}$ & $\begin{array}{l}-0.036 \\
(0.015)\end{array}$ & $\begin{array}{l}-0.025 \\
(0.022)\end{array}$ & $\begin{array}{l}-0.049 \\
(0.012)\end{array}$ & $\begin{array}{l}-0.037 \\
(0.017)\end{array}$ & $\begin{array}{l}-0.073 \\
(0.026)\end{array}$ & $\begin{array}{l}-0.044 \\
(0.022)\end{array}$ \\
\hline $\mathrm{P}$-value WB & 0.125 & 0.112 & 0.064 & 0.244 & 0.011 & 0.135 & 0.148 & 0.732 \\
\hline R-squared & 0.51 & 0.53 & 0.48 & 0.48 & 0.71 & 0.51 & 0.51 & 0.51 \\
\hline Observations & 240 & 240 & 240 & 225 & 240 & 234 & 240 & 240 \\
\hline \multicolumn{9}{|c|}{ IV - Old Informants } \\
\hline Espionage & $\begin{array}{l}-0.059 \\
(0.028)\end{array}$ & $\begin{array}{l}-0.111 \\
(0.052)\end{array}$ & $\begin{array}{l}-0.064 \\
(0.033)\end{array}$ & $\begin{array}{c}0.002 \\
(0.047)\end{array}$ & $\begin{array}{l}-0.004 \\
(0.032)\end{array}$ & $\begin{array}{l}-0.059 \\
(0.030)\end{array}$ & $\begin{array}{l}-0.092 \\
(0.041)\end{array}$ & $\begin{array}{l}-0.067 \\
(0.030)\end{array}$ \\
\hline $\mathrm{P}$-value WB & 0.353 & 0.264 & 0.173 & 0.978 & 0.927 & 0.372 & 0.339 & 0.327 \\
\hline F-stat & 60.5 & 58.8 & 19.0 & 28.1 & 16.4 & 56.2 & 25.4 & 19.6 \\
\hline Observations & 240 & 240 & 240 & 225 & 240 & 234 & 240 & 240 \\
\hline \multicolumn{9}{|c|}{ IV - Exit Informants } \\
\hline Espionage & $\begin{array}{l}-0.119 \\
(0.040)\end{array}$ & $\begin{array}{l}-0.170 \\
(0.073)\end{array}$ & $\begin{array}{l}-0.115 \\
(0.042)\end{array}$ & $\begin{array}{l}-0.181 \\
(0.109)\end{array}$ & $\begin{array}{l}-0.270 \\
(0.084)\end{array}$ & $\begin{array}{l}-0.116 \\
(0.039)\end{array}$ & $\begin{array}{l}-0.272 \\
(0.095)\end{array}$ & $\begin{array}{l}-0.120 \\
(0.038)\end{array}$ \\
\hline $\mathrm{P}$-value WB & 0.188 & 0.214 & 0.073 & 0.095 & 0.006 & 0.172 & 0.188 & 0.123 \\
\hline F-stat & 72.6 & 60.6 & 30.1 & 4.9 & 13.6 & 69.0 & 36.5 & 29.8 \\
\hline Observations & 192 & 192 & 192 & 180 & 192 & 189 & 192 & 192 \\
\hline
\end{tabular}

Note: Sample based on overlapping observations for the period 1970 to 1989. All regressions include time- and sectorspecific fixed effects, the patents gap and the initial log output per worker gap as additional regressors. Observations are weighted by the average number of workers in a sector (apart from columns (2) and (3)). The dependent variable is the change in the log output per worker gap between West and East Germany over the period $t$ to $t+x$, where $x \in\{1,3,5\}$. Column (1) restates our main results from column (6) of Table 2. In column (2), observations are weighted by the average sector-specific gross value added. In column (3), observations are unweighted. In column (4), we exclude the IT sector from the estimation sample. In column (5), we include sector-specific linear time trends in the specification. In column (6), we include the gap in the sector-specific import/output ratio between West and East Germany as an additional control variable. In column (7), we weight each piece of information according to the number of categorized keywords assigned to each sector. In column (8), we use machine learning methods to assign pieces of information to industry sectors. Standard errors are clustered at the sectoral level and shown in parentheses. P-value WB denotes p-values, relating to the espionage estimate, from Cameron et al. (2008)'s wild cluster bootstrap-t procedure using 999 replications. 
TABle A8: Robustness - 1-YeAR Intervals

\begin{tabular}{|c|c|c|c|c|c|c|c|c|}
\hline & $\begin{array}{l}\text { Main } \\
\text { spec }\end{array}$ & $\begin{array}{c}\text { Weighted } \\
\text { by output }\end{array}$ & $\begin{array}{c}\text { No } \\
\text { weights }\end{array}$ & $\begin{array}{l}\text { No } \\
\text { IT }\end{array}$ & $\begin{array}{l}\text { Sector } \\
\text { trends }\end{array}$ & $\begin{array}{c}\text { Trade } \\
\text { gap }\end{array}$ & $\begin{array}{l}\text { Keyword } \\
\text { weighted }\end{array}$ & $\begin{array}{l}\text { Machine } \\
\text { learning }\end{array}$ \\
\hline & $(1)$ & $(2)$ & $(3)$ & $(4)$ & $(5)$ & $(6)$ & $(7)$ & $(8)$ \\
\hline & \multicolumn{8}{|c|}{ Log TFP } \\
\hline \multicolumn{9}{|l|}{$\underline{\text { OLS }}$} \\
\hline Espionage & $\begin{array}{l}-0.032 \\
(0.009)\end{array}$ & $\begin{array}{l}-0.051 \\
(0.018)\end{array}$ & $\begin{array}{l}-0.029 \\
(0.012)\end{array}$ & $\begin{array}{l}-0.026 \\
(0.020)\end{array}$ & $\begin{array}{l}-0.033 \\
(0.009)\end{array}$ & $\begin{array}{l}-0.031 \\
(0.009)\end{array}$ & $\begin{array}{l}-0.052 \\
(0.009)\end{array}$ & $\begin{array}{l}-0.051 \\
(0.009)\end{array}$ \\
\hline P-value WB & 0.110 & 0.154 & 0.127 & 0.199 & 0.122 & 0.162 & 0.069 & 0.121 \\
\hline R-squared & 0.24 & 0.28 & 0.19 & 0.23 & 0.36 & 0.24 & 0.24 & 0.25 \\
\hline Observations & 304 & 304 & 304 & 285 & 304 & 298 & 304 & 304 \\
\hline \multicolumn{9}{|c|}{ IV - Old Informants } \\
\hline Espionage & $\begin{array}{l}-0.063 \\
(0.022)\end{array}$ & $\begin{array}{l}-0.109 \\
(0.048)\end{array}$ & $\begin{array}{l}-0.075 \\
(0.038)\end{array}$ & $\begin{array}{l}-0.072 \\
(0.064)\end{array}$ & $\begin{array}{l}-0.022 \\
(0.010)\end{array}$ & $\begin{array}{c}-0.055 \\
(0.019)\end{array}$ & $\begin{array}{l}-0.104 \\
(0.043)\end{array}$ & $\begin{array}{l}-0.064 \\
(0.026)\end{array}$ \\
\hline P-value WB & 0.113 & 0.190 & 0.192 & 0.326 & 0.542 & 0.124 & 0.194 & 0.204 \\
\hline F-stat & 116.8 & 159.1 & 87.0 & 64.6 & 196.3 & 106.9 & 18.9 & 18.8 \\
\hline Observations & 304 & 304 & 304 & 285 & 304 & 298 & 304 & 304 \\
\hline \multicolumn{9}{|c|}{ IV - Exit of Informants } \\
\hline Espionage & $\begin{array}{c}0.102 \\
(0.066)\end{array}$ & $\begin{array}{c}0.079 \\
(0.087)\end{array}$ & $\begin{array}{c}0.108 \\
(0.205)\end{array}$ & $\begin{array}{l}-0.136 \\
(0.150)\end{array}$ & $\begin{array}{c}0.153 \\
(0.065)\end{array}$ & $\begin{array}{c}0.104 \\
(0.066)\end{array}$ & $\begin{array}{c}0.162 \\
(0.091)\end{array}$ & $\begin{array}{c}0.102 \\
(0.055)\end{array}$ \\
\hline P-value WB & 0.328 & 0.531 & 0.439 & 0.207 & 0.312 & 0.296 & 0.251 & 0.241 \\
\hline F-stat & 5.6 & 3.3 & 0.4 & 1.8 & 5.9 & 5.7 & 11.2 & 12.6 \\
\hline \multirow[t]{2}{*}{ Observations } & 288 & 288 & 288 & 270 & 288 & 283 & 288 & 288 \\
\hline & \multicolumn{8}{|c|}{ Log Output per Worker } \\
\hline \multicolumn{9}{|l|}{$\underline{\text { OLS }}$} \\
\hline Espionage & $\begin{array}{l}-0.031 \\
(0.012)\end{array}$ & $\begin{array}{l}-0.046 \\
(0.019)\end{array}$ & $\begin{array}{l}-0.024 \\
(0.013)\end{array}$ & $\begin{array}{l}-0.013 \\
(0.015)\end{array}$ & $\begin{array}{l}-0.045 \\
(0.009)\end{array}$ & $\begin{array}{l}-0.030 \\
(0.012)\end{array}$ & $\begin{array}{l}-0.058 \\
(0.009)\end{array}$ & $\begin{array}{l}-0.055 \\
(0.008)\end{array}$ \\
\hline P-value WB & 0.397 & 0.220 & 0.162 & 0.374 & 0.077 & 0.436 & 0.089 & 0.143 \\
\hline R-squared & 0.24 & 0.32 & 0.20 & 0.23 & 0.34 & 0.24 & 0.25 & 0.26 \\
\hline Observations & 304 & 304 & 304 & 285 & 304 & 298 & 304 & 304 \\
\hline \multicolumn{9}{|c|}{ IV - Old Informants } \\
\hline Espionage & $\begin{array}{l}-0.049 \\
(0.019)\end{array}$ & $\begin{array}{l}-0.065 \\
(0.032)\end{array}$ & $\begin{array}{l}-0.045 \\
(0.036)\end{array}$ & $\begin{array}{c}0.005 \\
(0.051)\end{array}$ & $\begin{array}{l}-0.034 \\
(0.011)\end{array}$ & $\begin{array}{l}-0.040 \\
(0.018)\end{array}$ & $\begin{array}{l}-0.080 \\
(0.030)\end{array}$ & $\begin{array}{l}-0.049 \\
(0.018)\end{array}$ \\
\hline P-value WB & 0.326 & 0.284 & 0.470 & 0.944 & 0.641 & 0.391 & 0.264 & 0.291 \\
\hline F-stat & 103.3 & 148.2 & 81.7 & 75.6 & 192.7 & 94.2 & 18.9 & 19.2 \\
\hline Observations & 304 & 304 & 304 & 285 & 304 & 298 & 304 & 304 \\
\hline \multicolumn{9}{|c|}{ IV - Exit of Informants } \\
\hline Espionage & $\begin{array}{c}0.116 \\
(0.054)\end{array}$ & $\begin{array}{c}0.106 \\
(0.083)\end{array}$ & $\begin{array}{c}0.105 \\
(0.179)\end{array}$ & $\begin{array}{l}-0.050 \\
(0.144)\end{array}$ & $\begin{array}{c}0.154 \\
(0.066)\end{array}$ & $\begin{array}{c}0.120 \\
(0.054)\end{array}$ & $\begin{array}{c}0.188 \\
(0.076)\end{array}$ & $\begin{array}{c}0.119 \\
(0.046)\end{array}$ \\
\hline P-value WB & 0.205 & 0.480 & 0.428 & 0.633 & 0.322 & 0.178 & 0.180 & 0.147 \\
\hline F-stat & 6.2 & 3.5 & 0.5 & 1.8 & 5.8 & 6.2 & 12.4 & 13.5 \\
\hline Observations & 288 & 288 & 288 & 270 & 288 & 283 & 288 & 288 \\
\hline
\end{tabular}

Note: Sample based on annual intervals for the period 1970 to 1989. All regressions include time- and sector-specific fixed effects, the patents gap and the initial log TFP gap (upper panel) or log output per worker gap (lower panel) as additional regressors. Observations are weighted by the average number of workers in a sector (apart from columns (2) and (3)). The dependent variable is the change in the log TFP gap (upper panel) or log output per worker gap (lower panel) between West and East Germany over the period $t$ to $t+1$. The instrumental variables are described in Section V A. Column (1) restates our main results from column (3) of Table 2. In column (2), observations are weighted by the average sector-specific gross value added. In column (3), observations are unweighted. In column (4), we exclude the IT sector from the estimation sample. In column (5), we include sector-specific linear time trends in the specification. In column (6), we include the gap in the sector-specific import/output ratio between West and East Germany as an additional control variable. In column (7), we weight each piece of information according to the number of categorized keywords assigned to each sector. In column (8), we use machine learning methods to assign pieces of information to industry sectors. Standard errors are clustered at the sectoral level and shown in parentheses. P-value WB denotes p-values, relating to the espionage estimate, from Cameron et al. (2008)'s wild cluster bootstrap-t procedure using 999 replications. 
TABle A9: Robustness - 5-Year Intervals

\begin{tabular}{|c|c|c|c|c|c|c|c|c|}
\hline & $\begin{array}{l}\text { Main } \\
\text { spec }\end{array}$ & $\begin{array}{l}\text { Weighted } \\
\text { by output }\end{array}$ & $\begin{array}{c}\text { No } \\
\text { weights }\end{array}$ & $\begin{array}{l}\text { No } \\
\text { IT }\end{array}$ & $\begin{array}{l}\text { Sector } \\
\text { trends }\end{array}$ & $\begin{array}{l}\text { Trade } \\
\text { gap }\end{array}$ & $\begin{array}{l}\text { Keyword } \\
\text { weighted }\end{array}$ & $\begin{array}{l}\text { Machine } \\
\text { learning }\end{array}$ \\
\hline & $(1)$ & $(2)$ & $(3)$ & $(4)$ & $(5)$ & $(6)$ & $(7)$ & $(8)$ \\
\hline & \multicolumn{8}{|c|}{ Log TFP } \\
\hline \multicolumn{9}{|l|}{ OLS } \\
\hline Espionage & $\begin{array}{l}-0.034 \\
(0.022)\end{array}$ & $\begin{array}{l}-0.071 \\
(0.034)\end{array}$ & $\begin{array}{l}-0.029 \\
(0.014)\end{array}$ & $\begin{array}{l}-0.008 \\
(0.022)\end{array}$ & $\begin{array}{l}-0.012 \\
(0.028)\end{array}$ & $\begin{array}{l}-0.032 \\
(0.021)\end{array}$ & $\begin{array}{l}-0.084 \\
(0.029)\end{array}$ & $\begin{array}{l}-0.049 \\
(0.022)\end{array}$ \\
\hline P-value WB & 0.301 & 0.116 & 0.139 & 0.688 & 0.721 & 0.337 & 0.270 & 0.547 \\
\hline R-squared & 0.73 & 0.68 & 0.74 & 0.72 & 0.75 & 0.73 & 0.74 & 0.73 \\
\hline Observations & 176 & 176 & 176 & 165 & 176 & 170 & 176 & 176 \\
\hline \multicolumn{9}{|c|}{ IV - Old Informants } \\
\hline Espionage & $\begin{array}{l}-0.075 \\
(0.025)\end{array}$ & $\begin{array}{l}-0.128 \\
(0.051)\end{array}$ & $\begin{array}{c}-0.069 \\
(0.030)\end{array}$ & $\begin{array}{l}-0.026 \\
(0.031)\end{array}$ & $\begin{array}{l}-0.158 \\
(0.055)\end{array}$ & $\begin{array}{l}-0.067 \\
(0.023)\end{array}$ & $\begin{array}{l}-0.110 \\
(0.031)\end{array}$ & $\begin{array}{l}-0.077 \\
(0.023)\end{array}$ \\
\hline P-value WB & 0.242 & 0.232 & 0.101 & 0.452 & 0.024 & 0.333 & 0.119 & 0.156 \\
\hline F-stat & 49.0 & 33.9 & 12.7 & 19.8 & 5.7 & 45.7 & 28.3 & 41.1 \\
\hline Observations & 176 & 176 & 176 & 165 & 176 & 170 & 176 & 176 \\
\hline \multicolumn{9}{|c|}{ IV - Exit of Informants } \\
\hline Espionage & $\begin{array}{c}-9.628 \\
(609.036)\end{array}$ & $\begin{array}{c}1.254 \\
(3.125)\end{array}$ & $\begin{array}{c}0.654 \\
(2.943)\end{array}$ & $\begin{array}{l}-0.258 \\
(0.516)\end{array}$ & $\begin{array}{c}0.134 \\
(0.166)\end{array}$ & $\begin{array}{l}-0.466 \\
(2.167)\end{array}$ & $\begin{array}{c}0.515 \\
(1.138)\end{array}$ & $\begin{array}{l}-0.200 \\
(0.277)\end{array}$ \\
\hline $\mathrm{P}$-value WB & 0.984 & 0.556 & 0.683 & 0.429 & 0.412 & 0.696 & 0.454 & 0.382 \\
\hline F-stat & 0.0 & 0.1 & 0.0 & 0.3 & 1.7 & 0.0 & 0.3 & 1.6 \\
\hline \multirow[t]{2}{*}{ Observations } & 96 & 96 & 96 & 90 & 96 & 95 & 96 & 96 \\
\hline & \multicolumn{8}{|c|}{ Log Output per Worker } \\
\hline \multicolumn{9}{|l|}{ OLS } \\
\hline Espionage & $\begin{array}{l}-0.018 \\
(0.025)\end{array}$ & $\begin{array}{l}-0.045 \\
(0.039)\end{array}$ & $\begin{array}{l}-0.010 \\
(0.016)\end{array}$ & $\begin{array}{c}0.016 \\
(0.025)\end{array}$ & $\begin{array}{l}-0.005 \\
(0.026)\end{array}$ & $\begin{array}{l}-0.016 \\
(0.024)\end{array}$ & $\begin{array}{l}-0.066 \\
(0.032)\end{array}$ & $\begin{array}{l}-0.040 \\
(0.027)\end{array}$ \\
\hline P-value WB & 0.688 & 0.394 & 0.539 & 0.534 & 0.824 & 0.729 & 0.517 & 0.736 \\
\hline R-squared & 0.69 & 0.68 & 0.69 & 0.68 & 0.75 & 0.69 & 0.70 & 0.70 \\
\hline Observations & 176 & 176 & 176 & 165 & 176 & 170 & 176 & 176 \\
\hline \multicolumn{9}{|c|}{ IV - Old Informants } \\
\hline Espionage & $\begin{array}{l}-0.063 \\
(0.028)\end{array}$ & $\begin{array}{l}-0.111 \\
(0.054)\end{array}$ & $\begin{array}{l}-0.060 \\
(0.033)\end{array}$ & $\begin{array}{c}0.006 \\
(0.038)\end{array}$ & $\begin{array}{l}-0.150 \\
(0.058)\end{array}$ & $\begin{array}{l}-0.055 \\
(0.026)\end{array}$ & $\begin{array}{l}-0.093 \\
(0.035)\end{array}$ & $\begin{array}{l}-0.065 \\
(0.026)\end{array}$ \\
\hline $\mathrm{P}$-value WB & 0.460 & 0.314 & 0.220 & 0.906 & 0.023 & 0.578 & 0.303 & 0.377 \\
\hline F-stat & 46.5 & 35.4 & 10.7 & 15.5 & 5.6 & 43.8 & 29.7 & 44.0 \\
\hline Observations & 176 & 176 & 176 & 165 & 176 & 170 & 176 & 176 \\
\hline \multicolumn{9}{|c|}{ IV - Exit of Informants } \\
\hline Espionage & $\begin{array}{c}4.897 \\
(164.032)\end{array}$ & $\begin{array}{c}1.429 \\
(3.616)\end{array}$ & $\begin{array}{c}0.214 \\
(0.776)\end{array}$ & $\begin{array}{l}-0.109 \\
(0.427)\end{array}$ & $\begin{array}{c}0.125 \\
(0.223)\end{array}$ & $\begin{array}{l}-0.647 \\
(3.903)\end{array}$ & $\begin{array}{c}0.492 \\
(1.157)\end{array}$ & $\begin{array}{l}-0.189 \\
(0.274)\end{array}$ \\
\hline $\mathrm{P}$-value WB & 0.963 & 0.522 & 0.609 & 0.681 & 0.619 & 0.774 & 0.524 & 0.412 \\
\hline F-stat & 0.0 & 0.1 & 0.1 & 0.2 & 1.5 & 0.0 & 0.4 & 1.7 \\
\hline Observations & 96 & 96 & 96 & 90 & 96 & 95 & 96 & 96 \\
\hline
\end{tabular}

Note: Sample based on 5-year intervals and overlapping observations for the period 1970 to 1989. All regressions include time- and sector-specific fixed effects, the patents gap and the initial log TFP gap (upper panel) or log output per worker gap (lower panel) as additional regressors. Observations are weighted by the average number of workers in a sector (apart from columns (2) and (3)). The dependent variable is the change in the log TFP gap (upper panel) or log output per worker gap (lower panel) between West and East Germany over the period t to $t+5$. The instrumental variables are described in Section V A. Column (1) restates our main results from column (3) of Table 2. In column (2), observations are weighted by the average sector-specific gross value added. In column (3), observations are unweighted. In column (4), we exclude the IT sector from the estimation sample. In column (5), we include sector-specific linear time trends in the specification. In column (6), we include the gap in the sector-specific import/output ratio between West and East Germany as an additional control variable. In column (7), we weight each piece of information according to the number of categorized keywords assigned to each sector. In column (8), we use machine learning methods to assign pieces of information to industry sectors. Standard errors are clustered at the sectoral level and shown in parentheses. P-value WB denotes p-values, relating to the espionage estimate, from Cameron et al. (2008)'s wild cluster bootstrap-t procedure using 999 replications. 


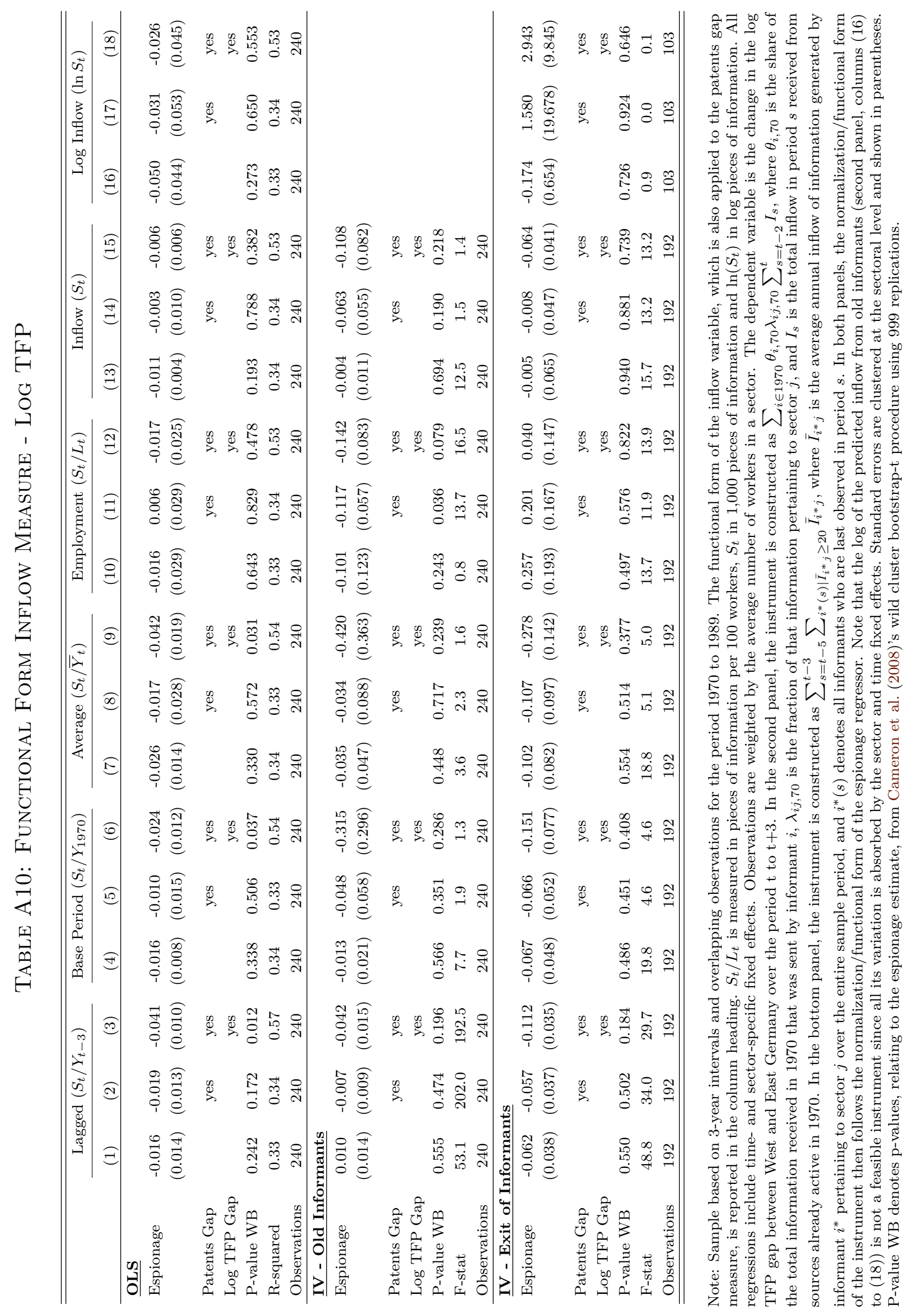




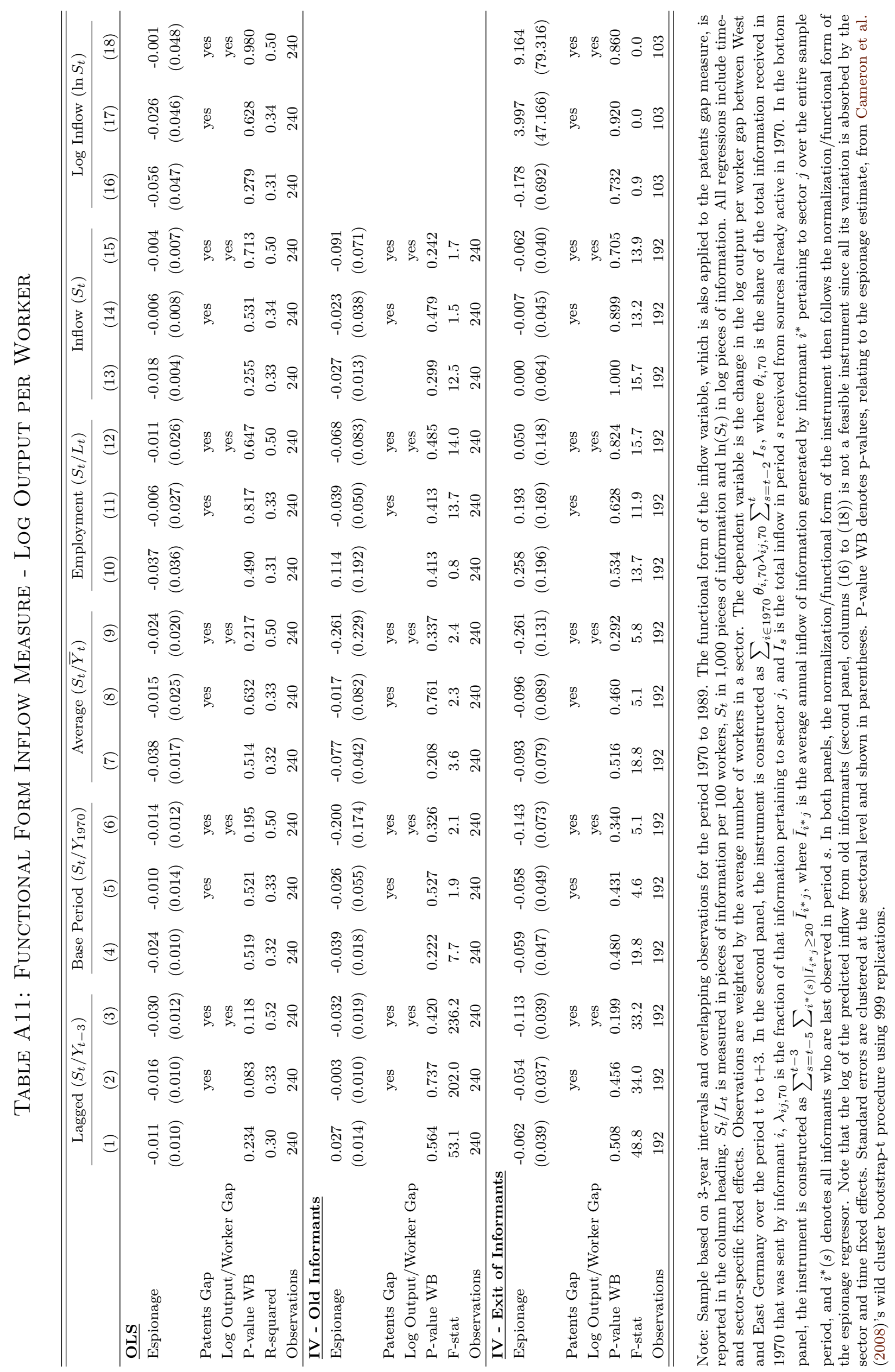


Table A12: Robustness - Construction of TFP Measures

\begin{tabular}{|c|c|c|c|c|c|}
\hline & \multicolumn{2}{|l|}{$\alpha$ flexible } & \multicolumn{3}{|c|}{$\alpha$ constant } \\
\hline & $\begin{array}{c}\text { Country/Industry } \\
(1)\end{array}$ & $\begin{array}{c}\text { Industry } \\
(2)\end{array}$ & $\begin{array}{c}\alpha=0.2 \\
(3)\end{array}$ & $\begin{array}{c}\alpha=0.33 \\
(4)\end{array}$ & $\begin{array}{c}\alpha=0.4 \\
(5)\end{array}$ \\
\hline \multicolumn{6}{|c|}{ Depreciation Rate 0.06} \\
\hline \multirow[t]{2}{*}{ Espionage } & -0.052 & -0.044 & -0.045 & -0.049 & -0.051 \\
\hline & $(0.012)$ & $(0.013)$ & $(0.014)$ & $(0.013)$ & $(0.012)$ \\
\hline \multirow[t]{2}{*}{ Patents Gap } & -0.038 & -0.020 & -0.011 & -0.024 & -0.030 \\
\hline & $(0.024)$ & $(0.026)$ & $(0.025)$ & $(0.022)$ & $(0.021)$ \\
\hline \multirow[t]{2}{*}{ Log TFP Gap } & -0.564 & -0.577 & -0.564 & -0.589 & -0.599 \\
\hline & $(0.090)$ & $(0.109)$ & $(0.101)$ & $(0.097)$ & $(0.094)$ \\
\hline $\mathrm{P}$-value WB & 0.011 & 0.050 & 0.055 & 0.027 & 0.028 \\
\hline R-squared & 0.56 & 0.54 & 0.53 & 0.55 & 0.56 \\
\hline Observations & 240 & 240 & 240 & 240 & 240 \\
\hline \multicolumn{6}{|c|}{ Depreciation Rate 0.02} \\
\hline \multirow[t]{2}{*}{ Espionage } & -0.049 & -0.042 & -0.042 & -0.044 & -0.046 \\
\hline & $(0.012)$ & $(0.013)$ & $(0.014)$ & $(0.013)$ & $(0.012)$ \\
\hline \multirow[t]{2}{*}{ Patents Gap } & -0.029 & -0.014 & -0.007 & -0.019 & -0.026 \\
\hline & $(0.023)$ & $(0.025)$ & $(0.025)$ & $(0.024)$ & $(0.023)$ \\
\hline \multirow[t]{2}{*}{ Log TFP Gap } & -0.525 & -0.546 & -0.546 & -0.567 & -0.578 \\
\hline & $(0.085)$ & $(0.104)$ & $(0.101)$ & $(0.101)$ & $(0.101)$ \\
\hline $\mathrm{P}$-value WB & 0.011 & 0.072 & 0.071 & 0.037 & 0.032 \\
\hline R-squared & 0.55 & 0.52 & 0.52 & 0.53 & 0.53 \\
\hline Observations & 240 & 240 & 240 & 240 & 240 \\
\hline \multicolumn{6}{|c|}{ Depreciation Rate 0.1} \\
\hline \multirow[t]{2}{*}{ Espionage } & -0.054 & -0.046 & -0.046 & -0.052 & -0.054 \\
\hline & $(0.012)$ & $(0.014)$ & $(0.015)$ & $(0.013)$ & $(0.012)$ \\
\hline \multirow[t]{2}{*}{ Patents Gap } & -0.048 & -0.028 & -0.015 & -0.030 & -0.036 \\
\hline & $(0.025)$ & $(0.026)$ & $(0.025)$ & $(0.022)$ & $(0.020)$ \\
\hline \multirow[t]{2}{*}{ Log TFP Gap } & -0.599 & -0.607 & -0.579 & -0.607 & -0.616 \\
\hline & $(0.096)$ & $(0.113)$ & $(0.100)$ & $(0.093)$ & $(0.087)$ \\
\hline $\mathrm{P}$-value WB & 0.009 & 0.040 & 0.050 & 0.024 & 0.013 \\
\hline R-squared & 0.57 & 0.54 & 0.54 & 0.57 & 0.58 \\
\hline Observations & 240 & 240 & 240 & 240 & 240 \\
\hline \multicolumn{6}{|c|}{ Depreciation Rate Flexible } \\
\hline \multirow[t]{2}{*}{ Espionage } & -0.051 & -0.043 & -0.044 & -0.047 & -0.049 \\
\hline & $(0.012)$ & $(0.013)$ & $(0.014)$ & $(0.013)$ & $(0.012)$ \\
\hline \multirow[t]{2}{*}{ Patents Gap } & -0.037 & -0.017 & -0.009 & -0.022 & -0.028 \\
\hline & $(0.023)$ & $(0.025)$ & $(0.025)$ & $(0.023)$ & $(0.022)$ \\
\hline \multirow[t]{2}{*}{ Log TFP Gap } & -0.556 & -0.564 & -0.557 & -0.580 & -0.591 \\
\hline & $(0.088)$ & $(0.107)$ & $(0.101)$ & $(0.099)$ & $(0.098)$ \\
\hline $\mathrm{P}$-value WB & 0.016 & 0.056 & 0.049 & 0.031 & 0.036 \\
\hline R-squared & 0.56 & 0.53 & 0.53 & 0.54 & 0.55 \\
\hline Observations & 240 & 240 & 240 & 240 & 240 \\
\hline
\end{tabular}

Note: Estimated specification as in column (3) of Table 2. In column (1), the technology parameters $\alpha_{i j}$ are sectorand country-specific. For West Germany, we use the average sector-specific capital shares over the period 1970 to 1989 reported in the EU KLEMS Growth and Productivity Accounts. For East Germany, we use the aggregate sector-specific capital shares of the ten Central and Eastern European countries that joined the EU in May 2004. In column (2), we use West German sector-specific capital shares to proxy for the the technology parameters $\alpha_{i j}$ in East Germany. In columns (3) to (5), we assume constant values for $\alpha_{i j}$ in both countries and across all sectors. In the first three panels, depreciation rates are assumed to be constant in both countries and across all sectors. In the bottom panel, we allow the depreciation rate to differ between West and East Germany. For West Germany, we use the average annual depreciation rate of the capital stock reported in the Penn World Table 9.0 for the period 1970 to $1989(4.2 \%)$. For East Germany, we use the unweighted average of all depreciation rates that pertain to Central and Eastern European countries, averaged over the period 1970 to 1989 (5.1\%). Standard errors are clustered at the sectoral level and shown in parentheses. P-value WB denotes p-values, relating to the espionage estimate, from Cameron et al. (2008)'s wild cluster bootstrap-t procedure using 999 replications. 
Table A13: Robustness - Construction of TFP Measures - IV Old

\begin{tabular}{|c|c|c|c|c|c|}
\hline & \multicolumn{2}{|l|}{$\alpha$ flexible } & \multicolumn{3}{|c|}{$\alpha$ constant } \\
\hline & $\begin{array}{c}\text { Country/Industry } \\
\text { (1) }\end{array}$ & $\begin{array}{c}\text { Industry } \\
(2)\end{array}$ & $\begin{array}{c}\alpha=0.2 \\
(3)\end{array}$ & $\begin{array}{c}\alpha=0.33 \\
(4)\end{array}$ & $\begin{array}{c}\alpha=0.4 \\
(5)\end{array}$ \\
\hline \multicolumn{6}{|c|}{ Depreciation Rate 0.06} \\
\hline \multirow[t]{2}{*}{ Espionage } & -0.072 & -0.060 & -0.063 & -0.066 & -0.067 \\
\hline & $(0.024)$ & $(0.025)$ & $(0.026)$ & $(0.025)$ & $(0.024)$ \\
\hline \multirow[t]{2}{*}{ Patents Gap } & -0.034 & -0.017 & -0.006 & -0.020 & -0.026 \\
\hline & $(0.024)$ & $(0.023)$ & $(0.023)$ & $(0.022)$ & $(0.021)$ \\
\hline \multirow[t]{2}{*}{ Log TFP Gap } & -0.571 & -0.580 & -0.565 & -0.591 & -0.601 \\
\hline & $(0.094)$ & $(0.106)$ & $(0.098)$ & $(0.095)$ & $(0.092)$ \\
\hline $\mathrm{P}$-value WB & 0.150 & 0.285 & 0.269 & 0.231 & 0.225 \\
\hline F-stat & 61.4 & 61.1 & 61.3 & 61.3 & 61.1 \\
\hline Observations & 240 & 240 & 240 & 240 & 240 \\
\hline \multicolumn{6}{|c|}{ Depreciation Rate 0.02} \\
\hline \multirow[t]{2}{*}{ Espionage } & -0.065 & -0.055 & -0.059 & -0.060 & -0.060 \\
\hline & $(0.024)$ & $(0.025)$ & $(0.026)$ & $(0.025)$ & $(0.025)$ \\
\hline \multirow[t]{2}{*}{ Patents Gap } & -0.026 & -0.010 & -0.002 & -0.015 & -0.022 \\
\hline & $(0.022)$ & $(0.022)$ & $(0.023)$ & $(0.022)$ & $(0.021)$ \\
\hline \multirow[t]{2}{*}{ Log TFP Gap } & -0.530 & -0.547 & -0.546 & -0.567 & -0.578 \\
\hline & $(0.088)$ & $(0.099)$ & $(0.097)$ & $(0.097)$ & $(0.096)$ \\
\hline $\mathrm{P}$-value WB & 0.188 & 0.318 & 0.305 & 0.274 & 0.273 \\
\hline F-stat & 61.8 & 61.7 & 61.5 & 62.0 & 62.2 \\
\hline Observations & 240 & 240 & 240 & 240 & 240 \\
\hline \multicolumn{6}{|c|}{ Depreciation Rate 0.1} \\
\hline \multirow[t]{2}{*}{ Espionage } & -0.080 & -0.066 & -0.067 & -0.072 & -0.074 \\
\hline & $(0.024)$ & $(0.025)$ & $(0.026)$ & $(0.025)$ & $(0.024)$ \\
\hline \multirow[t]{2}{*}{ Patents Gap } & -0.044 & -0.023 & -0.010 & -0.025 & -0.032 \\
\hline & $(0.027)$ & $(0.025)$ & $(0.024)$ & $(0.022)$ & $(0.021)$ \\
\hline \multirow[t]{2}{*}{ Log TFP Gap } & -0.609 & -0.611 & -0.581 & -0.610 & -0.619 \\
\hline & $(0.101)$ & $(0.112)$ & $(0.100)$ & $(0.093)$ & $(0.087)$ \\
\hline $\mathrm{P}$-value WB & 0.127 & 0.265 & 0.277 & 0.211 & 0.163 \\
\hline F-stat & 60.9 & 60.4 & 60.9 & 60.4 & 60.0 \\
\hline Observations & 240 & 240 & 240 & 240 & 240 \\
\hline \multicolumn{6}{|c|}{ Depreciation Rate Flexible } \\
\hline \multirow[t]{2}{*}{ Espionage } & -0.071 & -0.058 & -0.061 & -0.063 & -0.064 \\
\hline & $(0.024)$ & $(0.025)$ & $(0.026)$ & $(0.025)$ & $(0.025)$ \\
\hline \multirow[t]{2}{*}{ Patents Gap } & -0.033 & -0.014 & -0.004 & -0.018 & -0.024 \\
\hline & $(0.023)$ & $(0.023)$ & $(0.023)$ & $(0.022)$ & $(0.021)$ \\
\hline \multirow[t]{2}{*}{ Log TFP Gap } & -0.563 & -0.565 & -0.557 & -0.581 & -0.592 \\
\hline & $(0.092)$ & $(0.103)$ & $(0.098)$ & $(0.096)$ & $(0.094)$ \\
\hline $\mathrm{P}$-value WB & 0.169 & 0.306 & 0.286 & 0.224 & 0.239 \\
\hline F-stat & 61.9 & 61.4 & 61.4 & 61.7 & 61.7 \\
\hline Observations & 240 & 240 & 240 & 240 & 240 \\
\hline
\end{tabular}

Note: Estimated specification as in column (2) of Table 3. The instrumental variable is described in Section V A. In column (1), the technology parameters $\alpha_{i j}$ are sector- and country-specific. For West Germany, we use the average sector-specific capital shares over the period 1970 to 1989 reported in the EU KLEMS Growth and Productivity Accounts. For East Germany, we use the aggregate sector-specific capital shares of the ten Central and Eastern European countries that joined the EU in May 2004. In column (2), we use West German sector-specific capital shares to proxy for the the technology parameters $\alpha_{i j}$ in East Germany. In columns (3) to (5), we assume constant values for $\alpha_{i j}$ in both countries and across all sectors. In the first three panels, depreciation rates are assumed to be constant in both countries and across all sectors. In the bottom panel, we allow the depreciation rate to differ between West and East Germany. For West Germany, we use the average annual depreciation rate of the capital stock reported in the Penn World Table 9.0 for the period 1970 to 1989 (4.2\%). For East Germany, we use the unweighted average of all depreciation rates that pertain to Central and Eastern European countries, averaged over the period 1970 to 1989 (5.1\%). Standard errors are clustered at the sectoral level and shown in parentheses. P-value WB denotes p-values, relating to the espionage estimate, from Cameron et al. (2008)'s wild cluster bootstrap-t procedure using 999 replications. 
Table A14: Robustness - Construction of TFP Measures - IV Exit

\begin{tabular}{|c|c|c|c|c|c|}
\hline & \multicolumn{2}{|l|}{$\alpha$ flexible } & \multicolumn{3}{|c|}{$\alpha$ constant } \\
\hline & $\begin{array}{c}\text { Country/Industry } \\
\text { (1) }\end{array}$ & $\begin{array}{c}\text { Industry } \\
(2)\end{array}$ & $\begin{array}{c}\alpha=0.2 \\
(3)\end{array}$ & $\begin{array}{c}\alpha=0.33 \\
(4)\end{array}$ & $\begin{array}{c}\alpha=0.4 \\
(5)\end{array}$ \\
\hline \multicolumn{6}{|c|}{ Depreciation Rate 0.06} \\
\hline \multirow[t]{2}{*}{ Espionage } & -0.120 & -0.120 & -0.121 & -0.121 & -0.121 \\
\hline & $(0.036)$ & $(0.038)$ & $(0.037)$ & $(0.035)$ & $(0.033)$ \\
\hline \multirow[t]{2}{*}{ Patents Gap } & -0.059 & -0.037 & -0.026 & -0.043 & -0.052 \\
\hline & $(0.048)$ & $(0.048)$ & $(0.049)$ & $(0.048)$ & $(0.047)$ \\
\hline \multirow[t]{2}{*}{ Log TFP Gap } & -0.679 & -0.680 & -0.667 & -0.701 & -0.717 \\
\hline & $(0.140)$ & $(0.150)$ & $(0.143)$ & $(0.147)$ & $(0.146)$ \\
\hline $\mathrm{P}$-value WB & 0.149 & 0.174 & 0.162 & 0.135 & 0.104 \\
\hline F-stat & 50.4 & 60.8 & 59.7 & 52.7 & 49.8 \\
\hline Observations & 192 & 192 & 192 & 192 & 192 \\
\hline \multicolumn{6}{|c|}{ Depreciation Rate 0.02} \\
\hline \multirow[t]{2}{*}{ Espionage } & -0.116 & -0.115 & -0.118 & -0.118 & -0.117 \\
\hline & $(0.036)$ & $(0.037)$ & $(0.037)$ & $(0.035)$ & $(0.034)$ \\
\hline \multirow[t]{2}{*}{ Patents Gap } & -0.048 & -0.030 & -0.022 & -0.036 & -0.044 \\
\hline & $(0.045)$ & $(0.046)$ & $(0.047)$ & $(0.046)$ & $(0.046)$ \\
\hline \multirow[t]{2}{*}{ Log TFP Gap } & -0.630 & -0.640 & -0.641 & -0.661 & -0.672 \\
\hline & $(0.127)$ & $(0.139)$ & $(0.137)$ & $(0.140)$ & $(0.141)$ \\
\hline $\mathrm{P}$-value $\mathrm{WB}$ & 0.151 & 0.175 & 0.155 & 0.144 & 0.130 \\
\hline F-stat & 55.6 & 65.9 & 65.4 & 60.8 & 58.5 \\
\hline Observations & 192 & 192 & 192 & 192 & 192 \\
\hline \multicolumn{6}{|c|}{ Depreciation Rate 0.1} \\
\hline \multirow[t]{2}{*}{ Espionage } & -0.125 & -0.125 & -0.124 & -0.125 & -0.125 \\
\hline & $(0.037)$ & $(0.039)$ & $(0.037)$ & $(0.035)$ & $(0.033)$ \\
\hline \multirow[t]{2}{*}{ Patents Gap } & -0.071 & -0.045 & -0.031 & -0.051 & -0.061 \\
\hline & $(0.052)$ & $(0.051)$ & $(0.050)$ & $(0.050)$ & $(0.049)$ \\
\hline \multirow[t]{2}{*}{ Log TFP Gap } & -0.727 & -0.718 & -0.689 & -0.737 & -0.758 \\
\hline & $(0.152)$ & $(0.159)$ & $(0.148)$ & $(0.151)$ & $(0.149)$ \\
\hline $\mathrm{P}$-value WB & 0.148 & 0.170 & 0.158 & 0.107 & 0.091 \\
\hline F-stat & 47.1 & 57.2 & 55.6 & 47.8 & 45.4 \\
\hline Observations & 192 & 192 & 192 & 192 & 192 \\
\hline \multicolumn{6}{|c|}{ Depreciation Rate Flexible } \\
\hline \multirow[t]{2}{*}{ Espionage } & -0.119 & -0.118 & -0.120 & -0.119 & -0.119 \\
\hline & $(0.036)$ & $(0.037)$ & $(0.037)$ & $(0.035)$ & $(0.033)$ \\
\hline \multirow[t]{2}{*}{ Patents Gap } & -0.058 & -0.034 & -0.024 & -0.040 & -0.049 \\
\hline & $(0.047)$ & $(0.047)$ & $(0.048)$ & $(0.047)$ & $(0.046)$ \\
\hline \multirow[t]{2}{*}{ Log TFP Gap } & -0.668 & -0.662 & -0.656 & -0.684 & -0.698 \\
\hline & $(0.136)$ & $(0.146)$ & $(0.141)$ & $(0.144)$ & $(0.145)$ \\
\hline $\mathrm{P}$-value WB & 0.134 & 0.154 & 0.162 & 0.128 & 0.140 \\
\hline F-stat & 51.5 & 62.8 & 62.0 & 55.8 & 53.1 \\
\hline Observations & 192 & 192 & 192 & 192 & 192 \\
\hline
\end{tabular}

Note: Estimated specification as in column (4) of Table 3. The instrumental variable is described in Section V A. In column (1), the technology parameters $\alpha_{i j}$ are sector- and country-specific. For West Germany, we use the average sector-specific capital shares over the period 1970 to 1989 reported in the EU KLEMS Growth and Productivity Accounts. For East Germany, we use the aggregate sector-specific capital shares of the ten Central and Eastern European countries that joined the EU in May 2004. In column (2), we use West German sector-specific capital shares to proxy for the the technology parameters $\alpha_{i j}$ in East Germany. In columns (3) to (5), we assume constant values for $\alpha_{i j}$ in both countries and across all sectors. In the first three panels, depreciation rates are assumed to be constant in both countries and across all sectors. In the bottom panel, we allow the depreciation rate to differ between West and East Germany. For West Germany, we use the average annual depreciation rate of the capital stock reported in the Penn World Table 9.0 for the period 1970 to 1989 (4.2\%). For East Germany, we use the unweighted average of all depreciation rates that pertain to Central and Eastern European countries, averaged over the period 1970 to 1989 (5.1\%). Standard errors are clustered at the sectoral level and shown in parentheses. P-value WB denotes p-values, relating to the espionage estimate, from Cameron et al. (2008)'s wild cluster bootstrap-t procedure using 999 replications. 


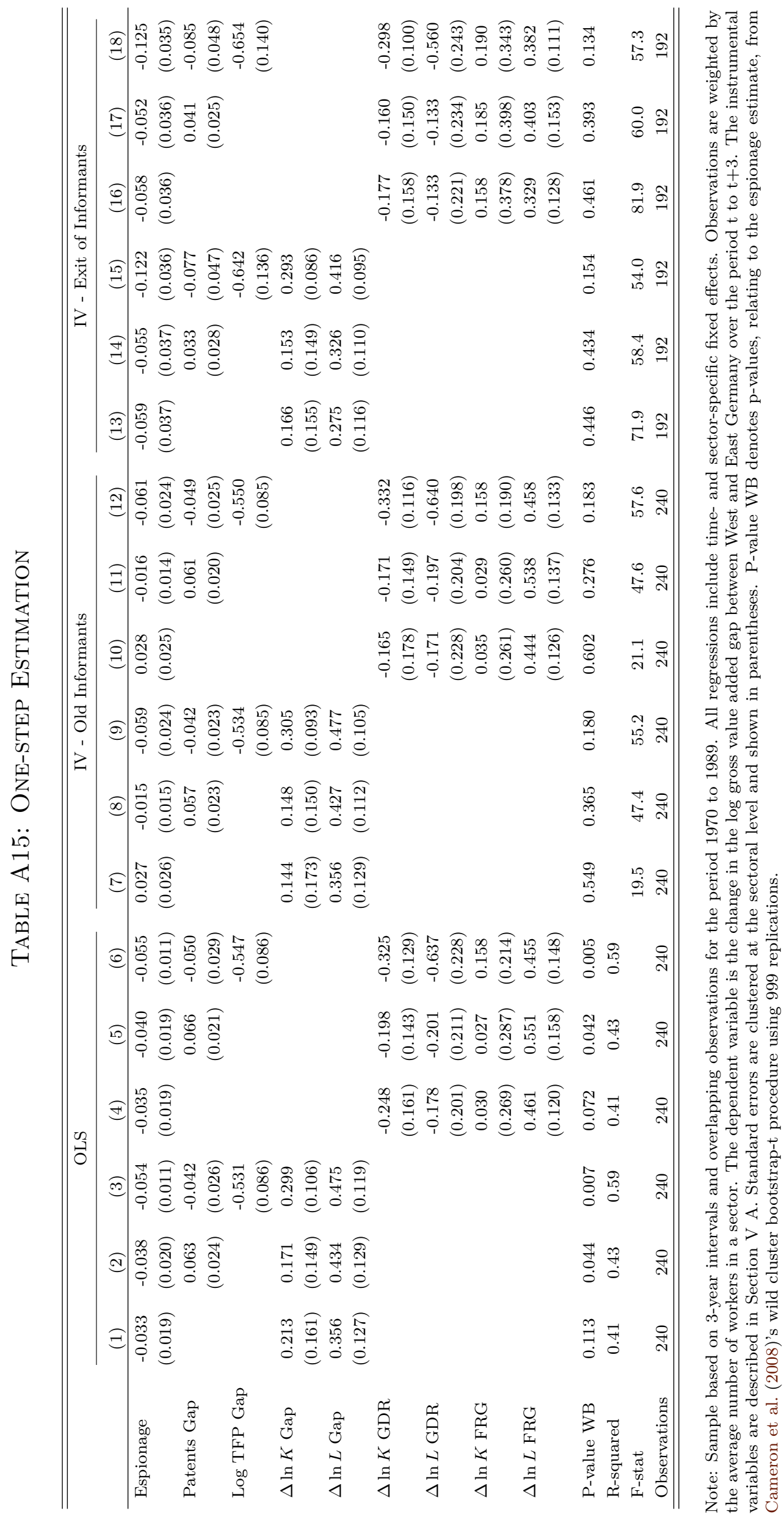


Table A16: Further Alternative Outcomes

\begin{tabular}{|c|c|c|}
\hline & GDR Exports & Patenting \\
\hline & (1) & $(2)$ \\
\hline \multicolumn{3}{|l|}{$\underline{\mathrm{OLS}}$} \\
\hline \multirow[t]{2}{*}{$\overline{\text { Espionage }}$} & 0.064 & -0.144 \\
\hline & $(0.029)$ & $(0.056)$ \\
\hline GDR Patents/Y & yes & yes \\
\hline Log TFP Gap & yes & yes \\
\hline $\mathrm{P}$-value WB & 0.176 & 0.047 \\
\hline R-squared & 0.04 & 0.98 \\
\hline Observations & 772 & 240 \\
\hline \multicolumn{3}{|c|}{ IV - Old Informants } \\
\hline \multirow[t]{2}{*}{ Espionage } & 0.212 & -0.236 \\
\hline & $(0.161)$ & $(0.027)$ \\
\hline GDR Patents/Y & yes & yes \\
\hline Log TFP Gap & yes & yes \\
\hline $\mathrm{P}$-value WB & 0.480 & 0.002 \\
\hline F-stat & 8.1 & 73.5 \\
\hline Observations & 772 & 240 \\
\hline \multicolumn{3}{|c|}{ IV - Exit of Informants } \\
\hline \multirow[t]{2}{*}{ Espionage } & -0.002 & -0.620 \\
\hline & $(0.108)$ & $(0.160)$ \\
\hline GDR Patents/Y & yes & yes \\
\hline Log TFP Gap & yes & yes \\
\hline $\mathrm{P}$-value WB & 0.980 & 0.182 \\
\hline F-stat & 10.5 & 32.4 \\
\hline Observations & 650 & 192 \\
\hline
\end{tabular}

Sample based on 3-year intervals and overlapping observations for the period 1970 to 1989. All regressions control for changes in the sector-specific log capital stock and employment as in equation (6), and include time- and sector-specific fixed effects. All dependent variables are measured as changes between period $t$ and $\mathrm{t}+3$. The instrumental variables are described in Section V.A. In column (1), the dependent variable is the change in the log value of East German exports of 86 distinct products compiled from East German Statistical Yearbooks (measured in nominal Valuta Mark). In column (2), the dependent variable is the future patent intensity in East Germany. Observations are weighted by the average number of workers in a sector. Standard errors are clustered at the sectoral level and shown in parentheses. P-value WB denotes p-values, relating to the espionage estimate, from Cameron et al. (2008)'s wild cluster bootstrap-t procedure using 999 replications. 


\section{Figures}

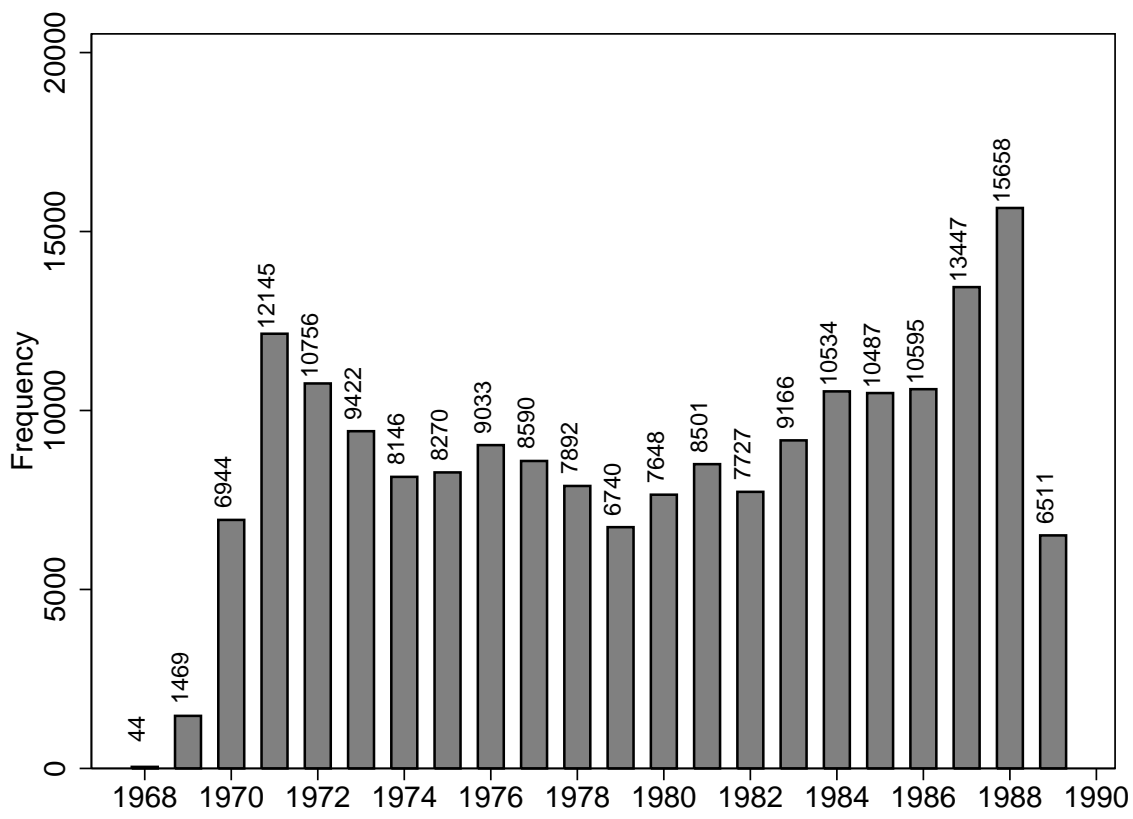

Figure A1: Information Inflow, 1968-1989

Note: Figure shows the annual inflow of information received by the HVA between 1968 and 1989. Data for 1968/69 and 1989 incomplete.

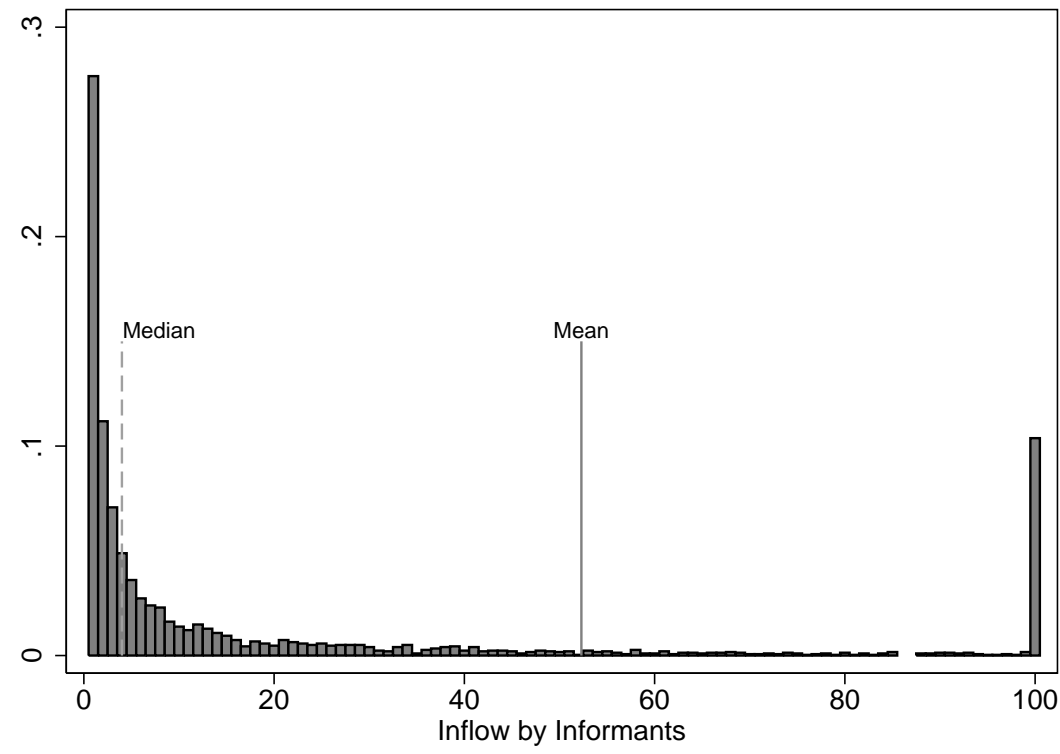

Figure A2: Inflow Distribution Across Informants

Note: The figure shows the distribution of the total number of pieces of information received from individual informants. For better readability, observations are censored at a value of 100 . 

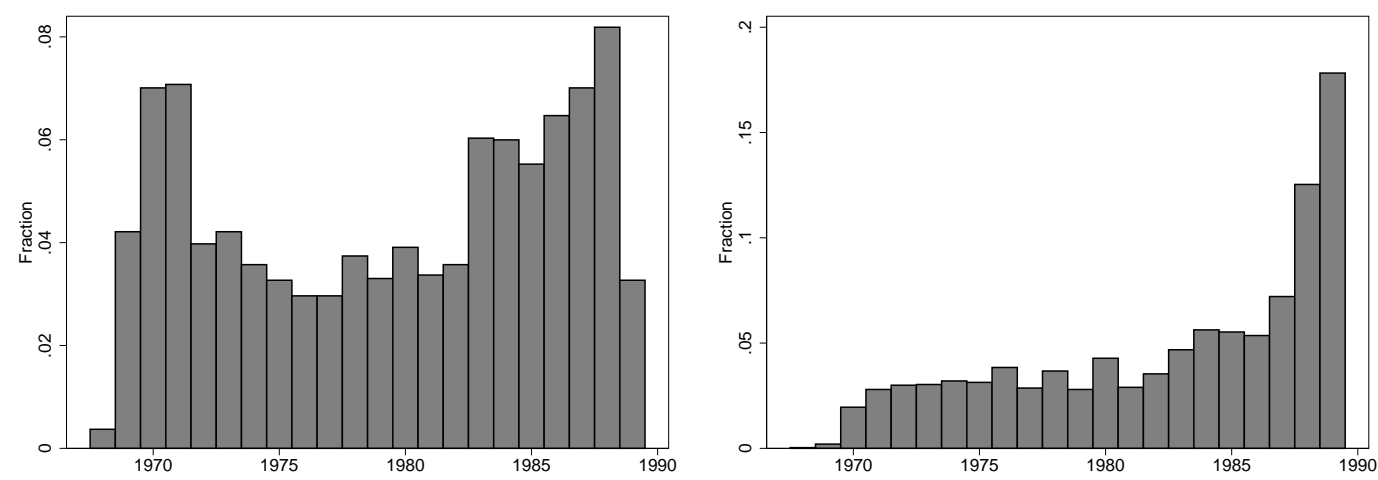

Figure A3: First and Last Active Year

Note: The figure shows the distributions of the first (left panel) and last year (right panel) in which individual informants are observed in the data. 

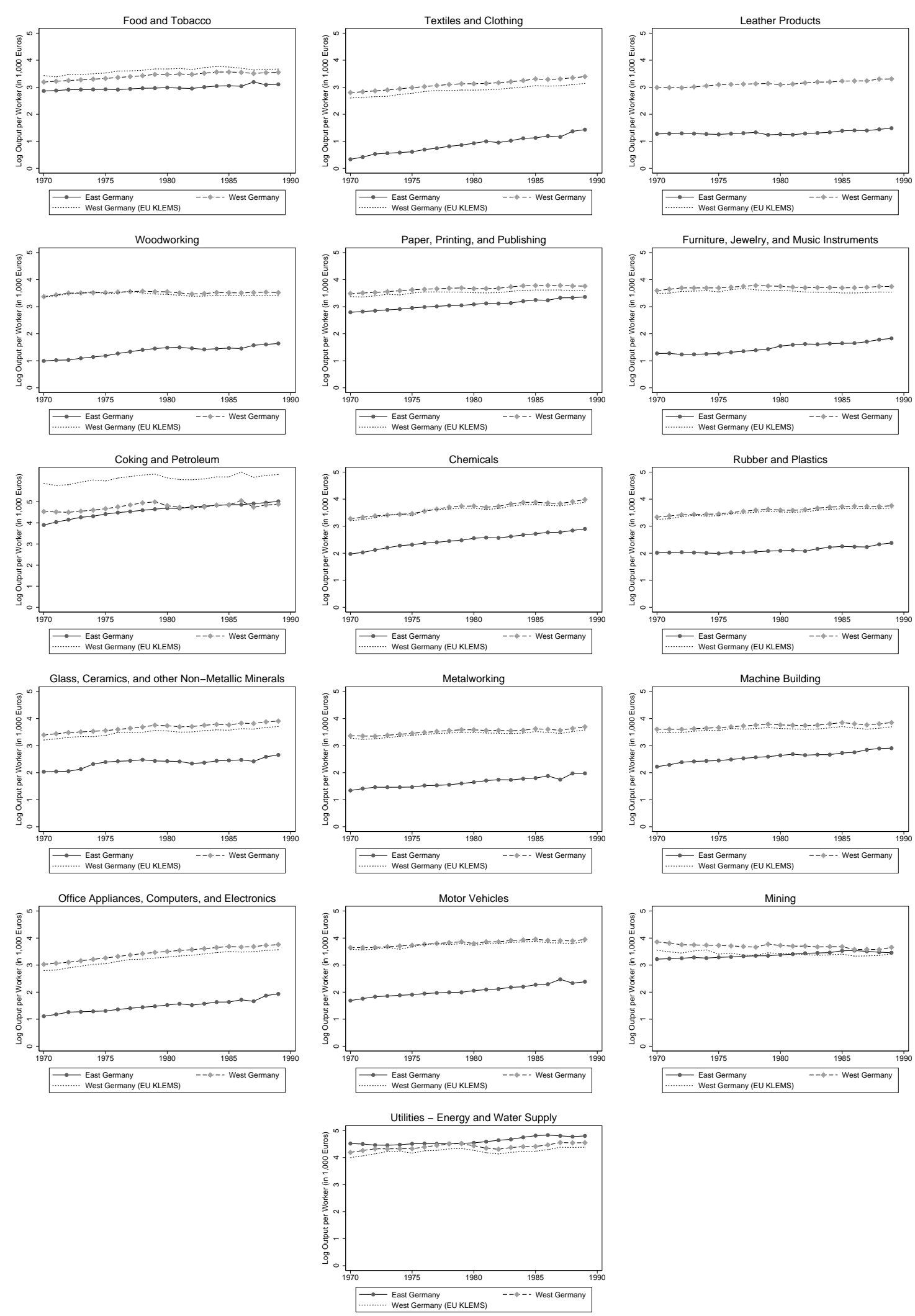

Figure A4: Log Output Per Worker By Sector

Note: The individual panels depict the log of gross value added per worker by sector for West and East Germany over the period 1970 to 1989. 

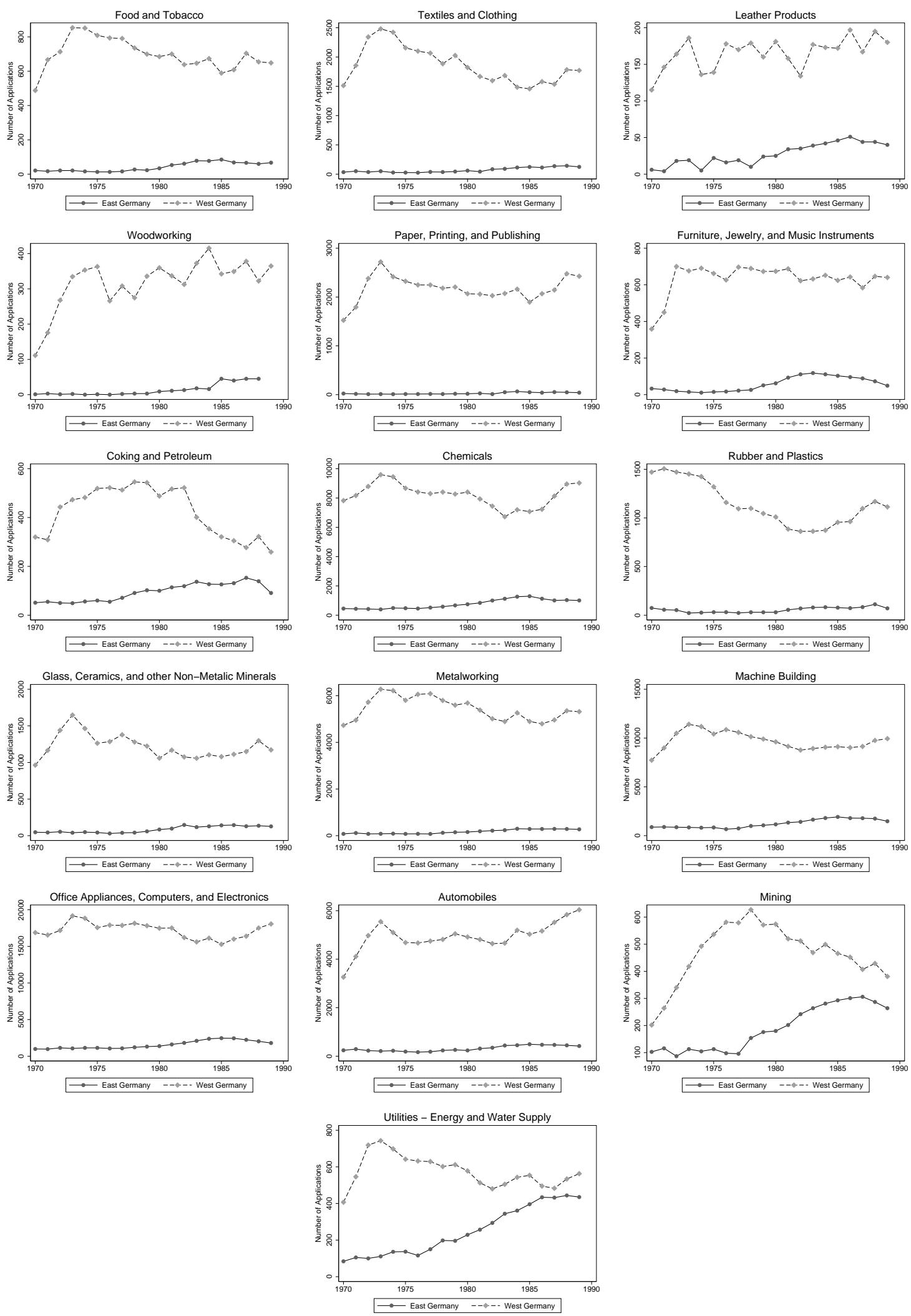

Figure A5: Patent Applications by Sector

Note: The individual panels depict the number of patent applications in West and East Germany for the corresponding sectors over the period 1970 to 1989. 

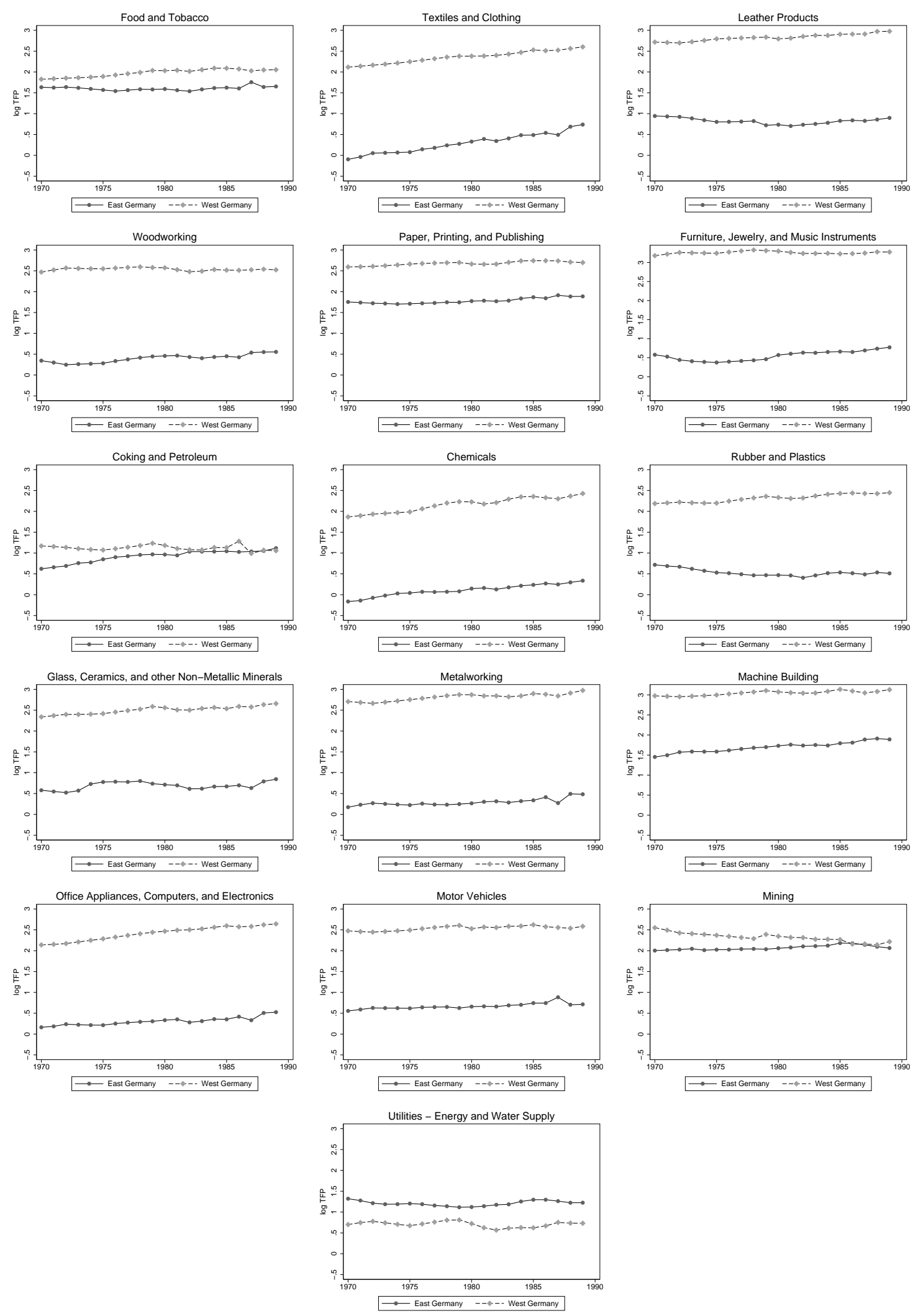

Figure A6: Log Total Factor Productivity by Sector

Note: The individual panels depict the estimated log TFP by sector for West and East Germany over the period 1970 to 1989 . TFP measures are constructed using the perpetual inventory as described in the text, assuming an annual depreciation rate of the capital stock of $6 \%$ and sector-specific capital shares taken from the EU KLEMS Growth and Productivity Accounts (November 2009 Release, updated March 2011). 

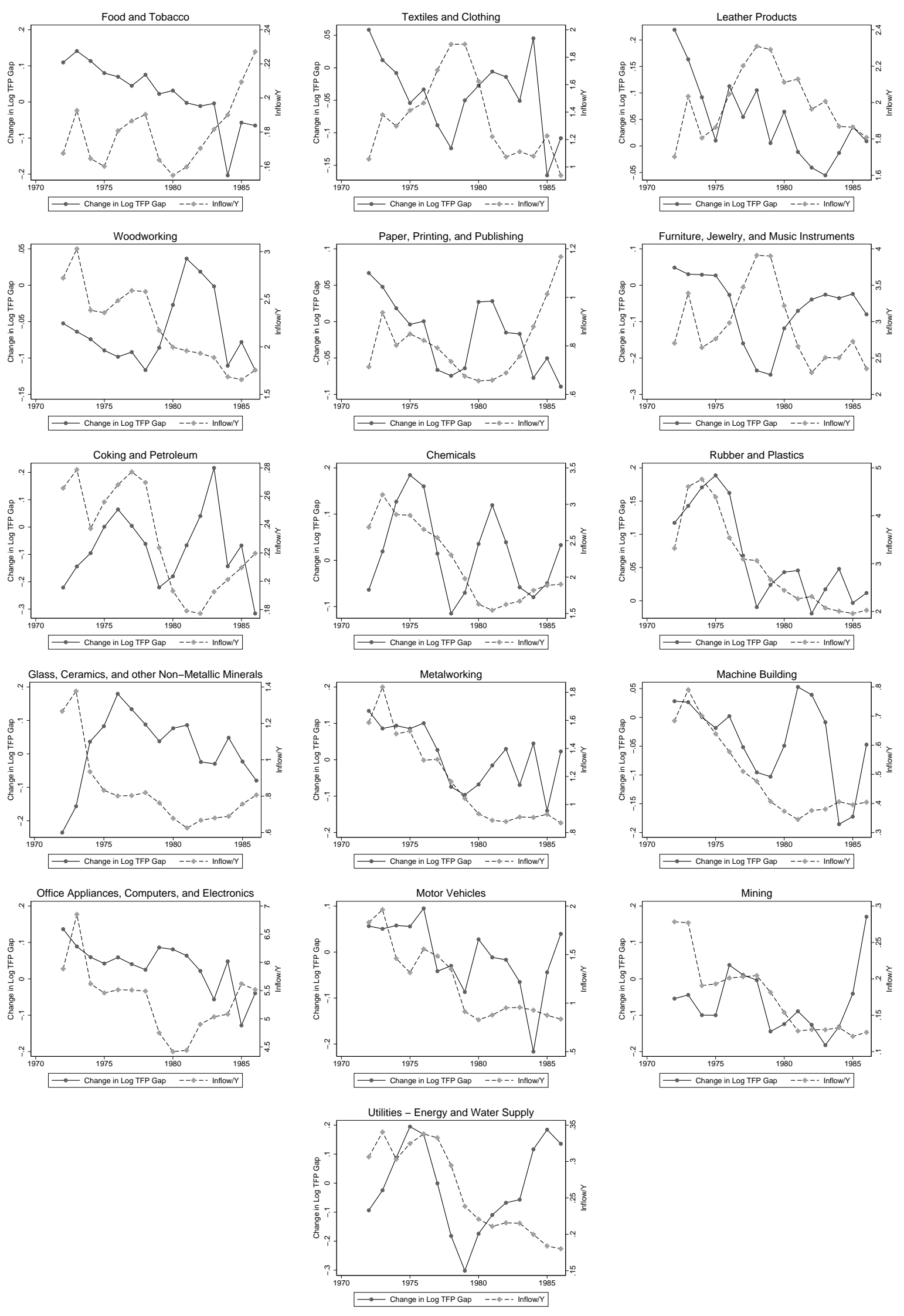

\section{Figure A7: Change in Log TFP Gap and Information Inflow}

Note: The individual panels depict for each sector the change in the log TFP gap between West and East Germany between $t$ and $t+3$ and the accumulated inflow of information scaled by output between $t-3$ and $t$. 

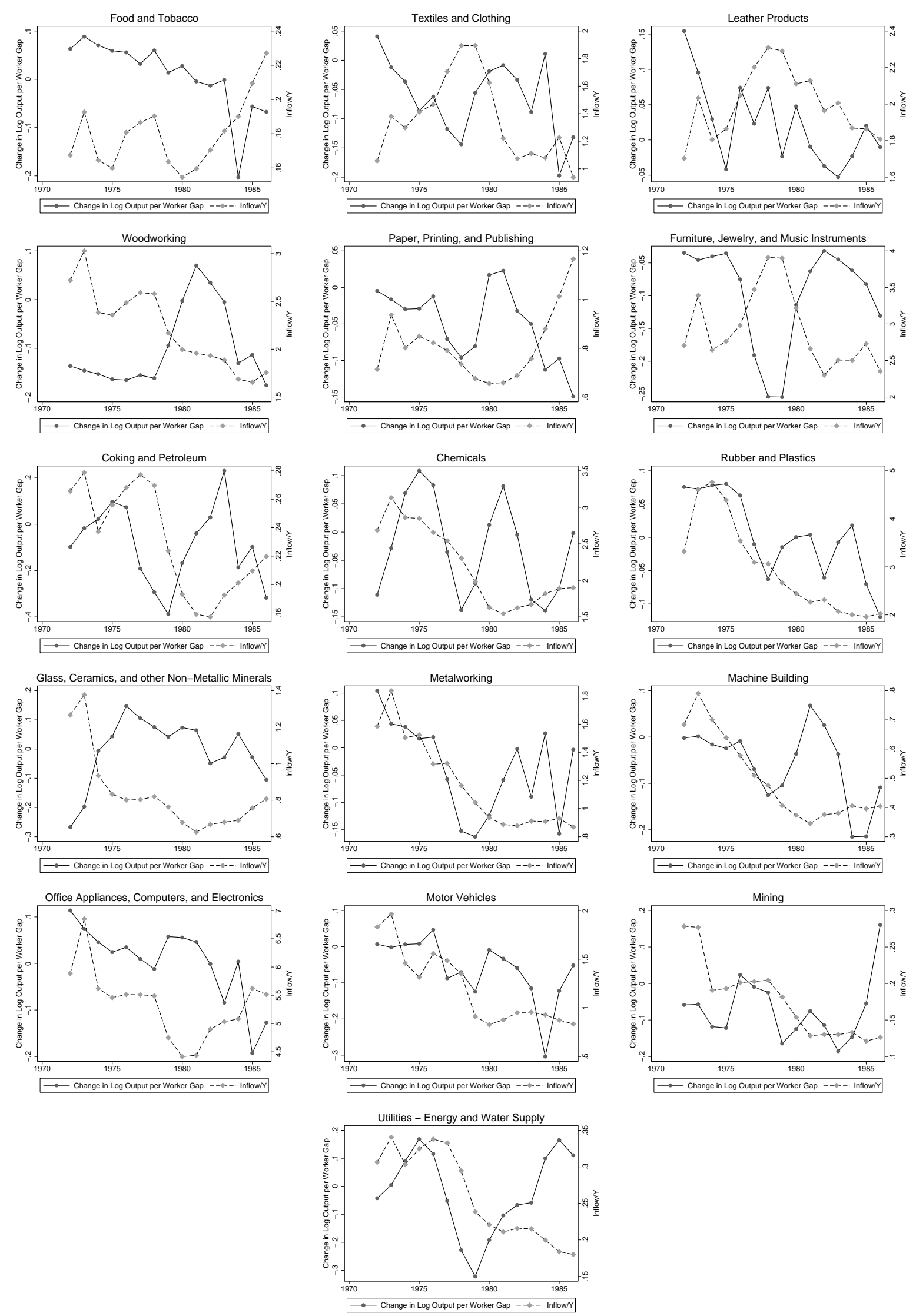

\section{Figure A8: Change in Log Output Per Worker Gap and Information Inflow}

Note: The individual panels depict for each sector the change in the log output per worker gap between West and East Germany between $t$ and $t+3$ and the accumulated inflow of information scaled by output between $t-3$ and $t$. 

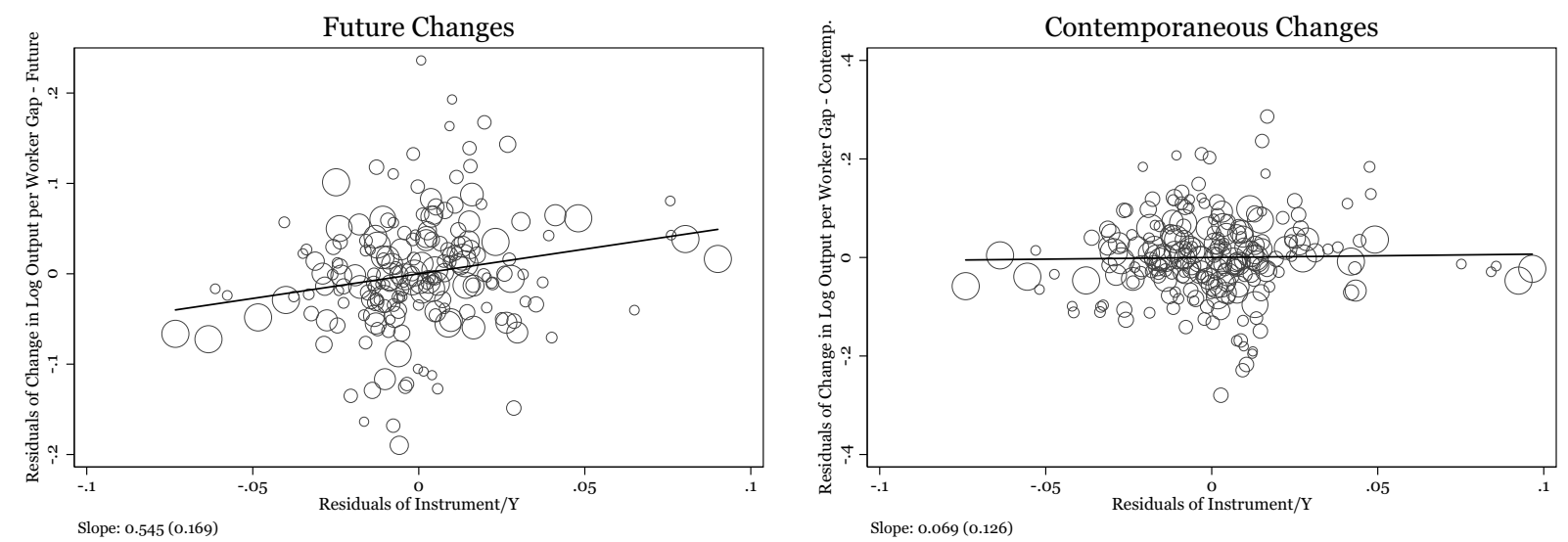

\section{Figure A9: Exits of Informants and Changes in the Log Output Per Worker GAP}

Note: The figure plots residualized changes in the log output per worker gap between West and East Germany against residualized exits of highly prolific informants scaled by output. Exits are measured between the end of period t- 6 and t-3. Changes in the log output per worker gap are measured between the end of period $t$ and $t+3$ in the left panel and the end of t-6 and t-3 in the right panel. Circles are proportional to the square root of the average number of workers in an industry. The solid black lines represent the OLS regression lines.
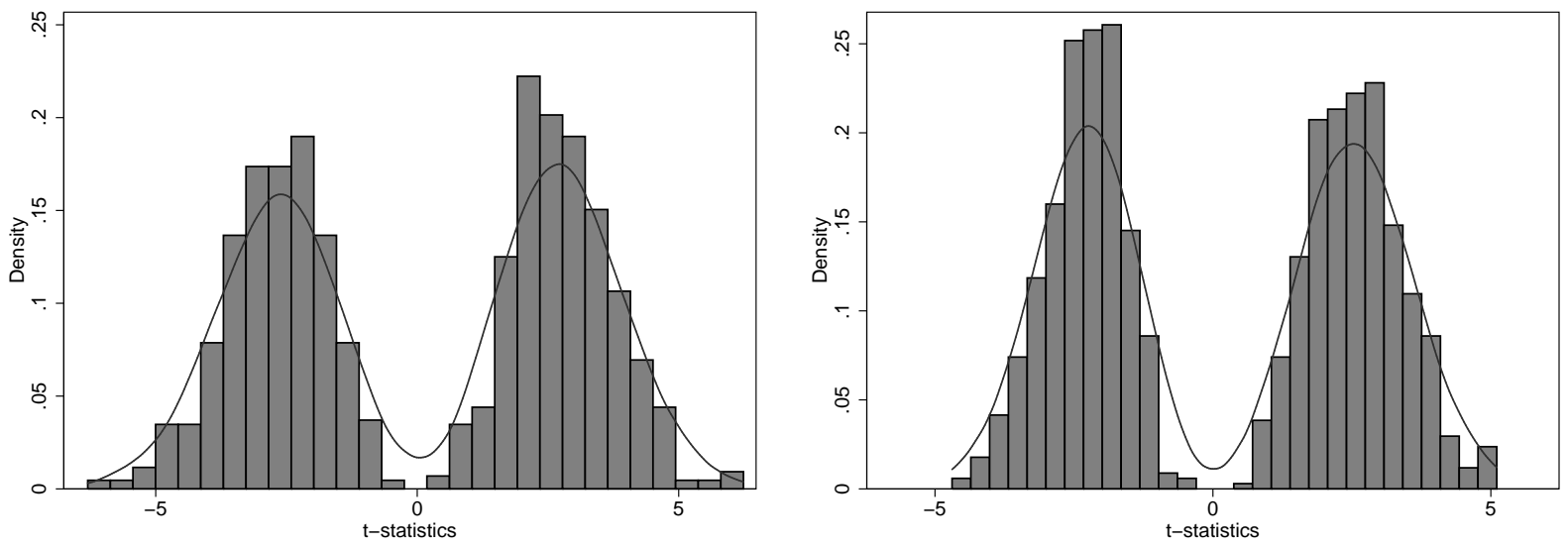

Figure A10: Distribution t-Statistics After Wild Cluster Bootstrap

Note: The left panel depicts the distribution of the bootstrap t-statistics underlying the WB p-values reported in column (8) of the top panel in Table 5. The right panel depicts the distribution of the bootstrap t-statistics underlying the WB p-values reported in column (3) of the bottom panel in Table A10. The plotted densities are based on an Epanechnikov kernel function with default bandwidth 0.667 and 0.584 respectively. 

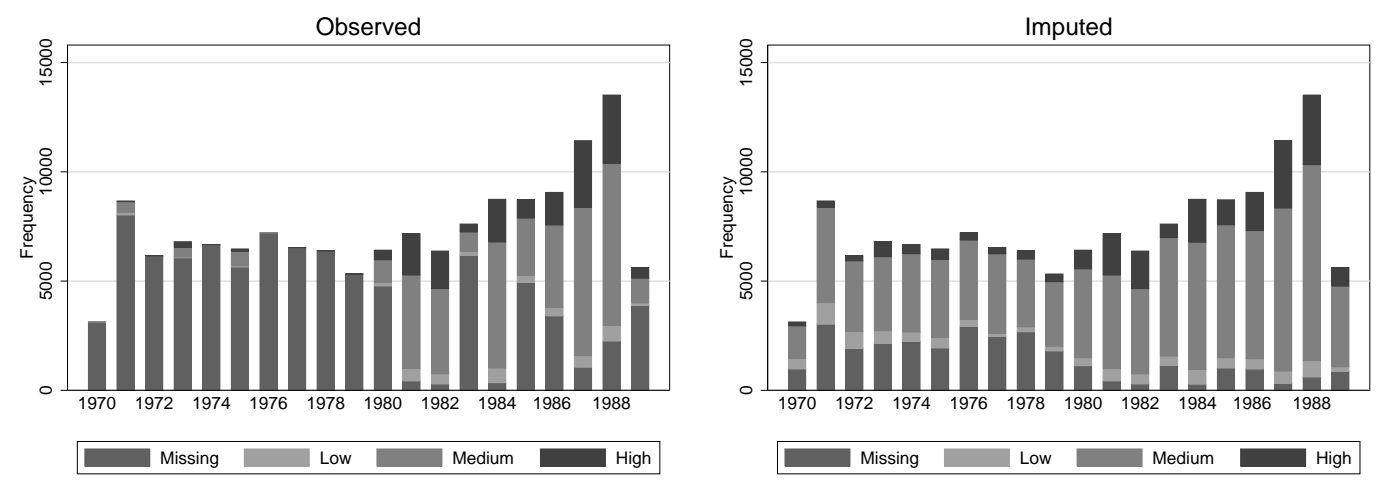

Figure A11: Distribution of Quality Assessments

Note: The figure shows the distribution of quality assessments by year, both as observed in the data (left panel) and after imputing missing observations using the experienced-adjusted expected quality assessments of the informant generating the information (right panel). "Low" comprises assessments of 4 and 5, "Medium" assessments of 3, and "High" comprises assessments of 1 and 2. 

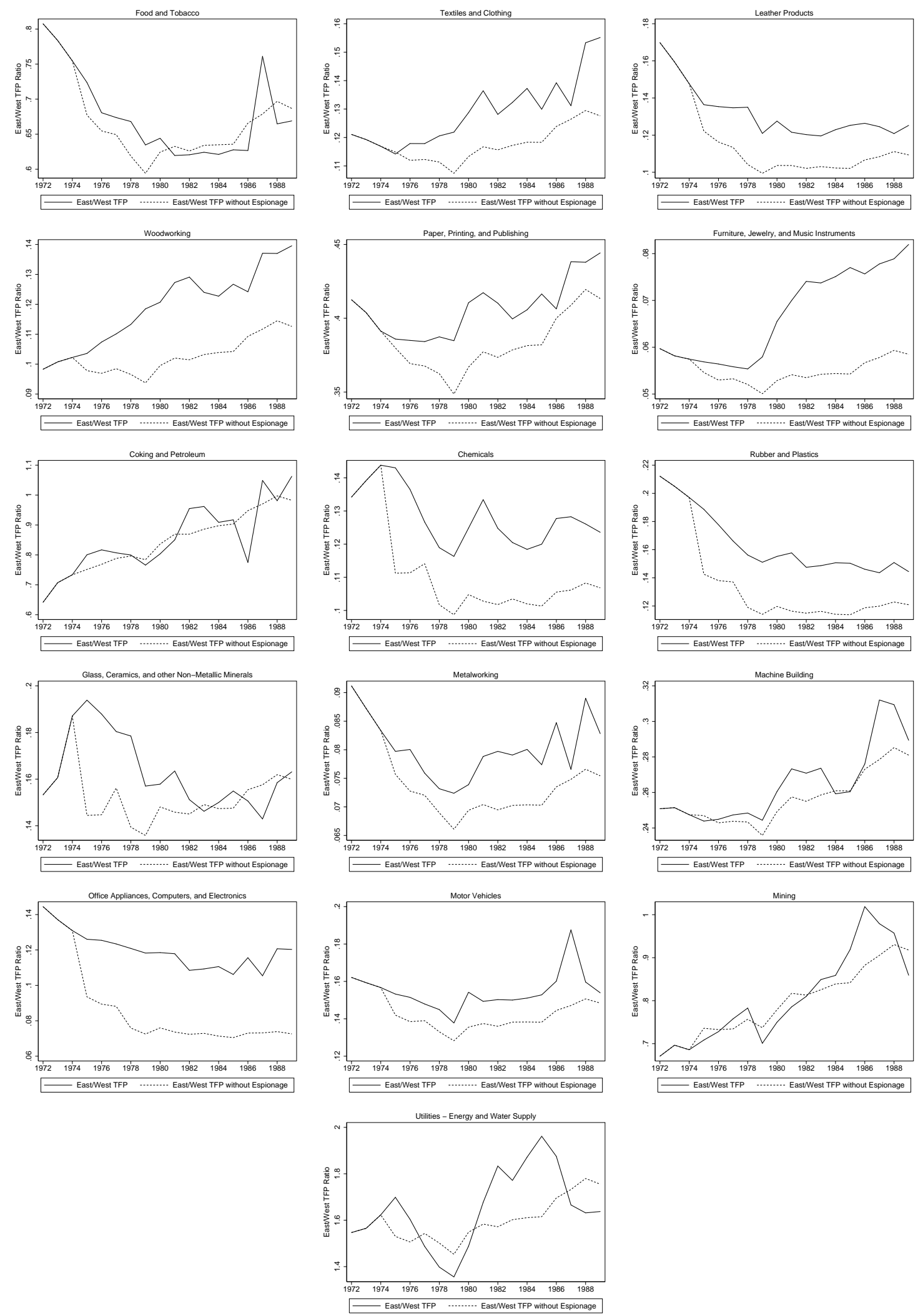

Figure A12: Counterfactual Simulations by Sector

Note: The individual panels depict the actual and counterfactual East/West TFP ratios in the corresponding sectors. The counterfactual simulations are based on the empirical results reported in column (3) of Table 2 . 


\section{References}

Cameron, A. Colin, Jonah B. Gelbach, and Douglas L. Miller, "Bootstrap-based Improvements for Inference with Clustered Standard Errors," The Review of Economics and Statistics, 2008, 90 (3), 414-427. 